\title{
Diffusion with nonlocal Robin boundary conditions
}

\author{
By Wolfgang Arendt, Stefan Kunkel and Markus Kunze
}

(Received Oct. 24, 2016)

(Revised Apr. 23, 2017)

\begin{abstract}
We investigate a second order elliptic differential operator $A_{\beta, \mu}$ on a bounded, open set $\Omega \subset \mathbb{R}^{d}$ with Lipschitz boundary subject to a nonlocal boundary condition of Robin type. More precisely we have $0 \leq \beta \in$ $L^{\infty}(\partial \Omega)$ and $\mu: \partial \Omega \rightarrow \mathscr{M}(\bar{\Omega})$, and boundary conditions of the form

$$
\partial_{\nu}^{\mathscr{A}} u(z)+\beta(z) u(z)=\int_{\bar{\Omega}} u(x) \mu(z)(\mathrm{d} x), \quad z \in \partial \Omega,
$$

where $\partial_{\nu}^{\mathscr{A}}$ denotes the weak conormal derivative with respect to our differential operator. Under suitable conditions on the coefficients of the differential operator and the function $\mu$ we show that $A_{\beta, \mu}$ generates a holomorphic semigroup $T_{\beta, \mu}$ on $L^{\infty}(\Omega)$ which enjoys the strong Feller property. In particular, it takes values in $C(\bar{\Omega})$. Its restriction to $C(\bar{\Omega})$ is strongly continuous and holomorphic. We also establish positivity and contractivity of the semigroup under additional assumptions and study the asymptotic behavior of the semigroup.
\end{abstract}

\section{Introduction.}

In the 1950s Feller [19], [20], [21] described all diffusion processes in one dimension; in particular, he characterized the boundary conditions which lead to generators of what today is called a Feller semigroup. Besides the classical Dirichlet, Neumann and Robin boundary conditions, also certain nonlocal boundary conditions can occur. In higher dimensions, it was Venttsel' [37] who first described the boundary conditions satisfied by the functions in the domain of the generator of a Feller semigroup. Naturally, the converse question of which of these boundary conditions actually lead to generators of Feller semigroups has recieved a lot of attention. The starting point for that question is the article by Sato and Ueno [32], who proved that this is the case if and only if a certain auxiliary problem (which is a generalization of the Dirichlet problem, involving the boundary condition in question; cf. Equation (3.1) below) is solvable for sufficiently many right-hand sides. Some concrete examples of boundary conditions for which one obtains a generator of a Feller semigroup were already contained in $[\mathbf{3 2}],[\mathbf{3 7}]$; more refined results were obtained by Taira, see [35] and the references therein, Skubachevskiı $[\mathbf{3 3}],[\mathbf{3 4}]$ and Galakhov and Skubachevskiu [22].

In this article, we are concerned with diffusion equations with certain non-local boundary conditions of Robin type. Let us describe this in more detail. We consider a bounded, open set $\Omega \subset \mathbb{R}^{d}$ with Lipschitz boundary. As far as our boundary condition is concerned, we make the following assumptions.

2010 Mathematics Subject Classification. Primary 47D07, 60J35, 35B35.

Key Words and Phrases. diffusion process, non-local boundary condition, stability. 
Hypothesis 1.1. We are given a real-valued function $0 \leq \beta \in L^{\infty}(\partial \Omega)$, where $\partial \Omega$ is endowed with surface measure $\sigma$. Moreover, we are given a map $\mu: \partial \Omega \rightarrow \mathscr{M}(\bar{\Omega})$, the space of complex-valued measures on $\bar{\Omega}$, which satisfies the following conditions.

(a) For every function $f \in B_{b}(\bar{\Omega})$, the space of all bounded and Borel measurable functions on $\bar{\Omega}$, the map $z \mapsto\langle f, \mu(z)\rangle:=\int_{\bar{\Omega}} f(x) \mu(z)(\mathrm{d} x)$ is measurable;

(b) for some $p>d-1$ with $p \geq 2$ we have $\int_{\partial \Omega}\|\mu(z)\|^{p} \mathrm{~d} \sigma(z)<\infty$ and

(c) there exists a positive and bounded measure $\tau$ on $\bar{\Omega}$ such that for every $z \in \partial \Omega$ the measure $\mu(z)$ is absolutely continuous with respect to $\tau$.

In (a), it actually suffices to assume that the map $z \mapsto\langle f, \mu(z)\rangle$ is measurable for all $f \in C(\bar{\Omega})$. The measurability for those $f$ which are merely bounded and measurable follows by a monotone class argument, cf. the proof of Lemma 6.1 in [25]. We will see later on that if instead of (a) we assume

$\left(\mathrm{a}^{\prime}\right)$ For every $f \in B_{b}(\bar{\Omega})$ the map $z \mapsto\langle f, \mu(z)\rangle$ is continuous

then parts (b) and (c) in Hypothesis 1.1 are automatically satisfied.

Assuming Hypothesis 1.1 we can define the operator $\Delta_{\beta, \mu}$ on $L^{\infty}(\Omega)$ by

$$
\begin{aligned}
& D\left(\Delta_{\beta, \mu}\right):=\left\{u \in H^{1}(\Omega) \cap C(\bar{\Omega}): \Delta u \in L^{\infty}(\Omega),\right. \\
&\left.\partial_{\nu} u(z)+\beta(z) u(z)=\langle u, \mu(z)\rangle \forall z \in \partial \Omega\right\}, \\
& \Delta_{\beta, \mu} u=\Delta u .
\end{aligned}
$$

Here $H^{1}(\Omega)$ is the usual Sobolev space and the normal derivative $\partial_{\nu} u$ has to be understood as follows.

Definition 1.2. For a function $u \in H^{1}(\Omega)$, we write $\operatorname{tr} u$ for its trace in $L^{2}(\partial \Omega)$. Let $u \in H^{1}(\Omega)$ be such that $\Delta u \in L^{2}(\Omega)$ and let $h \in L^{2}(\partial \Omega)$. We say that $\partial_{\nu} u=h$ if Green's formula

$$
\int_{\Omega} \Delta u v \mathrm{~d} x+\int_{\Omega} \nabla u \nabla v \mathrm{~d} x=\int_{\partial \Omega} h \operatorname{tr} v \mathrm{~d} \sigma
$$

holds for all $v \in H^{1}(\Omega)$.

In what follows we will not distinguish between a function $u \in H^{1}(\Omega)$ and its trace $\operatorname{tr} u$ in integrals over the boundary $\partial \Omega$.

With this definition of the normal derivative the operator $\Delta_{\beta, \mu}$ is well-defined. Indeed, if $u \in D\left(\Delta_{\beta, \mu}\right)$ then $u \in C(\bar{\Omega})$ whence

$$
h(z):=\langle u, \mu(z)\rangle-\beta(z) u(z)
$$

defines a function $h \in L^{2}(\partial \Omega)$. Since furthermore $u \in H^{1}(\Omega)$ and $\Delta u \in L^{\infty}(\Omega) \subset L^{2}(\Omega)$ it makes sense to say that $\partial_{\nu} u=h$. This condition is the Robin boundary condition we are interested in with local part $\beta \operatorname{tr} u$ and non-local part $\langle u, \mu(\cdot)\rangle$.

We also consider the part $\Delta_{\beta, \mu}^{C}$ of $\Delta_{\beta, \mu}$ in $C(\bar{\Omega})$ given by 


$$
\begin{gathered}
D\left(\Delta_{\beta, \mu}^{C}\right):=\left\{u \in H^{1}(\Omega) \cap C(\bar{\Omega}): \Delta u \in C(\bar{\Omega}),\right. \\
\left.\quad \partial_{\nu} u+\left.\beta u\right|_{\partial \Omega}=\langle u, \mu(\cdot)\rangle\right\}
\end{gathered}
$$

One of our main results is the following generation theorem.

THEOREM 1.3. Assuming Hypothesis 1.1, the operator $\Delta_{\beta, \mu}$ generates a holomorphic semigroup $\left(T_{\beta, \mu}(t)\right)_{t>0}$ on $L^{\infty}(\Omega)$ which satisfies the strong Feller property. In particular, this semigroup leaves the space $C(\bar{\Omega})$ invariant. Its restriction to $C(\bar{\Omega})$ is a strongly continuous and holomorphic semigroup whose generator is $\Delta_{\beta, \mu}^{C}$.

We refer to Section 2 for the definition of holomorphic semigroups which are not strongly continuous at 0 and for an explanation of the strong Feller property. We will actually prove Theorem 1.3 in more generality, replacing the Laplacian with a general second order strictly elliptic differential operator with measurable coefficients.

We will also establish positivity and contractivity of the semigroup $T_{\beta, \mu}$ under additional assumptions on $\beta$ and $\mu$, see Section 5. In the case of Theorem 1.3, where we consider the Laplacian, the conditions are as follows. If the measures $\mu(z)$ are positive for all $z \in \partial \Omega$ then the semigroup $T_{\beta, \mu}$ is positive; i.e. each $T_{\beta, \mu}(t)$ leaves the positive cone $L^{\infty}(\Omega)_{+}$of $L^{\infty}(\Omega)$ invariant. If additionally we have that

$$
\mu(z, \bar{\Omega}) \leq \beta(z) \text { for almost all } z \in \partial \Omega,
$$

then the semigroup $T_{\beta, \mu}$ is sub-Markovian, i.e. $T_{\beta, \mu}$ is positive and $T_{\beta, \mu}(t) \mathbb{1} \leq \mathbb{1}$ for all $t>0$. If equality holds in (1.1) then $T_{\beta, \mu}$ is Markovian, i.e. $T_{\beta, \mu}(t)$ is positive and $T_{\beta, \mu}(t) \mathbb{1}=\mathbb{1}$. In these situations we will also study the asymptotic behavior of the semigroup $T_{\beta, \mu}$. In the sub-Markovian, non-Markovian case the semigroup converges in operator norm to 0 , whereas in the Markovian case the orbits converge to an equilibrium.

Let us compare our results to the existing literature. First of all, in this article we consider less restrictive assumptions on the coefficients and the domain. Indeed, in the above mentioned references, the domain and the coefficients of the operator are assumed to be smooth (i.e. $C^{\infty}$ or a suitable Hölder continuity), whereas here we consider coefficients which are merely measurable and a domain with Lipschitz boundary. Moreover, we prove our generation result for general boundary conditions and study additional properties, such as positivity and the Markov property, afterwards, whereas in [33], [34], [22], $[35]$ there are a priori assumptions imposed on the coefficients in the boundary condition which ensure these properties. On the other hand, the quoted result treat more general boundary conditions which cover also, e.g., viscosity phenomena on the boundary.

Possibly the most important novelty in this article is that we obtain a holomorphic semigroup on $C(\bar{\Omega})$, even on $L^{\infty}(\Omega)$. So far, holomorphic semigroups for diffusion processes with nonlocal boundary conditions were only established on the $L^{p}$-scale $(1 \leq p<\infty)$, see [36]. To the best of our knowledge, the only other article which establishes holomorphy of the semigroup on $C(\bar{\Omega})$ for diffusion operators with non-local boundary conditions is our previous article [8], where we have treated non-local Dirichlet boundary conditions. We should note that the two problems are rather different. Indeed, 
the non-local Robin boundary condition considered here falls in the so-called 'transversal case', where, due to the normal derivative, the non-local term has lower order than the rest of the boundary condition. This is not the case for the non-local Dirichlet boundary condition which falls in the so-called 'non-transversal case'. Also the strategy for the proof is rather different. The proof of Theorem 1.3 is based on a perturbation result by Greiner, which we explain in Section 3. We will actually present a slight generalization of Greiner's result which establishes additional properties of the perturbed semigroup. We should mention that Greiner's perturbation result cannot be used in the case of non-local Dirichlet boundary conditions where the maximum principle plays an essential role.

The holomorphy of the semigroup together with the compactness allows us to study the asymptotic behavior of the semigroup in Section 6 .

Non-local Robin boundary conditions of the above form occur in several concrete situations, for example in heat control, where the heat is measured in the interior and the control is via the boundary, see $[\mathbf{1 3}],[\mathbf{2 4}]$.

The structure of this article is as follows. After some preliminaries in Section 2, we present Greiner's boundary perturbation, along with our modifications, in Section 3. Section 4 contains results on elliptic differential operators with local Robin boundary conditions which are needed subsequently. In Section 5 we prove our main generation result. Section 6 contains our results concerning the asymptotic behavior of the semigroup and Section 7 is devoted to the special situation where all measures $\mu(z)$ are absolutely continuous with respect to Lebesgue measure. There we will see that our conditions for positivity and sub-Markovianity are necessary in this situation. The concluding Section 8 contains some examples where Hypothesis 1.1 is satisfied, in particular, we prove that it is satisfied whenever condition $\left(a^{\prime}\right)$ is fulfilled. In the appendix we present some general results on the asymptotic behavior of positive semigroups, which we use in Section 6 .

\section{Preliminaries.}

\subsection{Semigroups that are not necessarily strongly continuous.}

In this article, we shall consider semigroups on the space $L^{\infty}(\Omega)$, where $\Omega$ is a bounded open subset of $\mathbb{R}^{d}$. By a result of Lotz [27] (see also [6, Corollary 4.3.19]), a strongly continuous semigroup on $L^{\infty}(\Omega)$ necessarily has a bounded generator. As we are concerned with second order differential operators, we will encounter semigroups that are not strongly continuous. Since this is not a standard situation, we recall the relevant definitions and results here. Let us start with the following definition, taken from $[\mathbf{6}$, Section 3.2].

Definition 2.1. Let $X$ be a Banach space. A semigroup is a strongly continuous mapping $T:(0, \infty) \rightarrow \mathscr{L}(X)$ such that

(a) $T(t+s)=T(t) T(s)$ for all $t, s>0$;

(b) there exist constants $M>0$ and $\omega \in \mathbb{R}$ such that $\|T(t)\| \leq M e^{\omega t}$ for all $t>0$;

(c) if $T(t) x=0$ for all $t>0$, it follows that $x=0$.

We say that $T$ is of type $(M, \omega)$ to emphasize that $(b)$ holds with these constants. A semigroup of type $(1,0)$ is called contraction semigroup. If additionally we have 


$$
T(t) x \rightarrow x \quad \text { as } \quad t \rightarrow 0
$$

for all $x \in X$, then $T$ is called strongly continuous.

Clearly, the condition $T(t) x \rightarrow x$ as $t \rightarrow 0$ for every $x \in X$ implies condition (c) above and it is not difficult to see that it also implies condition (b) (see [18, I, Proposition $5.5]$ ). Thus, our definition of strongly continuous semigroup coincides with the classical definition used, e.g., in [18, I, Definition 5.1]. However, even without strong continuity, we can associate a generator with a semigroup. Indeed, if $T$ is a semigroup of type $(M, \omega)$, then there exists a unique operator $G$ such that $(\omega, \infty)$ is contained in the resolvent set $\rho(G)$ of $G$ and

$$
R(\lambda, G) x=\int_{0}^{\infty} e^{-\lambda t} T(t) x d t
$$

for all $x \in X$ and $\lambda>\omega$, see [6, Equation (3.13)]. The operator $G$ is called the generator of $T$. Note that in the case of strongly continuous semigroups this is equivalent to the usual 'differential' definition of the generator, see [18, II, Theorem 1.10].

A semigroup $T$ is called holomorphic, if there is some angle $\theta \in(0, \pi / 2]$ such that $T$ has a holomorphic extension to the sector

$$
\Sigma_{\theta}:=\left\{r e^{i \varphi}: r>0,|\varphi|<\theta\right\}
$$

which is bounded on $\Sigma_{\theta} \cap\{z \in \mathbb{C}:|z| \leq 1\}$, see [6, Definition 3.7.1].

The generators of holomorphic semigroups can be characterized as follows.

TheOREM 2.2. An operator $G$ on $X$ generates a holomorphic semigroup if and only if there exists a constant $\omega \in \mathbb{R}$ such that $\{\lambda \in \mathbb{C}: \operatorname{Re} \lambda>\omega\} \subset \rho(G)$ and

$$
\sup _{\operatorname{Re} \lambda>\omega}\|\lambda R(\lambda, G)\|<\infty .
$$

Proof. [28, Proposition 2.1.11] or [6, Corollary 3.7.12 and Proposition 3.7.4].

The following Lemma is taken from [28, Proposition 2.1.4].

LEMma 2.3. Let $T$ be a holomorphic semigroup with generator $G$. Then we have $T(t) x \rightarrow x$ if and only if $x \in \overline{D(G)}$.

It follows from Lemma 2.3 that a holomorphic semigroup is strongly continuous if and only if its generator is densely defined. Recalling from [28, Proposition 2.1.1] that $D(G)$ (and hence also $\overline{D(G)}$ ) is invariant under $T$, a second corollary of Lemma 2.3 is that every holomorphic semigroup $T$ restricts to a strongly continuous and holomorphic semigroup on $\overline{D(G)}$.

\subsection{Transition kernels and the strong Feller property.}

In the study of Markov processes it is important that the transition semigroup consists of kernel operators, as these give the transition probabilities of the process. We recall the relevant definitions and results and introduce the strong Feller property which 
is important for the ergodic theory of Markov processes. In this subsection, $K$ is a compact metric space and $\mathscr{B}(K)$ denotes the Borel $\sigma$-algebra on $K$. Later on, we will consider $K=\bar{\Omega}$.

A (bounded) kernel on $K$ is a map $k: K \times \mathscr{B}(K) \rightarrow \mathbb{C}$ such that

(i) the map $x \mapsto k(x, A)$ is Borel-measurable for all $A \in \mathscr{B}(K)$,

(ii) the map $A \mapsto k(x, A)$ is a (complex) measure on $\mathscr{B}(K)$ for each $x \in K$ and

(iii) we have $\sup _{x \in K}|k|(x, K)<\infty$, where $|k|(x, \cdot)$ denotes the total variation of the measure $k(x, \cdot)$.

Let $X=C(K)$ or $X=B_{b}(K)$. We call an operator $T \in \mathscr{L}(X)$ a kernel operator if there exists a kernel $k$ such that

$$
T f(x)=\int_{K} f(y) k(x, d y)
$$

for all $f \in X$ and $x \in K$. As there is at most one kernel $k$ satisfying the above equation, we call $k$ the kernel associated with $T$. Conversely $T$ is called the operator associated with $k$.

Let us note that every bounded operator on $C(K)$ is a kernel operator, since given $T \in \mathscr{L}(X)$ we can set $k(x, \cdot):=T^{*} \delta_{x} \in \mathscr{M}(K)$ for every $x \in K$. Standard arguments (cf. [25, Proposition 3.5]) show that $k$ is indeed a kernel and it is then easy to see that $T$ is associated with $k$. On the other hand, not every bounded operator on $B_{b}(K)$ is a kernel operator. We have the following characterization.

Lemma 2.4. Let $T \in \mathscr{L}\left(B_{b}(K)\right)$. The following are equivalent.

(i) $T$ is a kernel operator.

(ii) $T$ is pointwise continuous, i.e. if $f_{n}$ is a bounded sequence converging pointwise to $f$, then $T f_{n}$ converges pointwise to $T f$.

Proof. The implication '(i) $\Rightarrow$ (ii)' follows from the dominated convergence theorem. For the converse, put $k(x, A):=\left(T \mathbb{1}_{A}\right)(x)$, where $\mathbb{1}_{A}$ denotes the indicator function of the set $A \in \mathscr{B}(K)$. Using (ii), we see that $k(x, \cdot)$ is a measure, thus $k$ is a kernel. By the density of simple functions in $B_{b}(K)$ with respect to the supremum norm, we easily see that $T$ is associated with $k$.

Let us note that given a kernel operator $T$ on $C(K)$, we can always extend $T$ to a kernel operator $\tilde{T}$ on $B_{b}(K)$ by defining $(\tilde{T} f)(x)$ by the right-hand side of $(2.1)$ for $f \in B_{b}(K)$. The operator $\tilde{T}$ is called the canonical extension of $T$. The operator $T$ may have other extensions to a bounded operator on $B_{b}(K)$, but $\tilde{T}$ is the only one which is a kernel operator.

Definition 2.5. A kernel operator $T$ on $B_{b}(K)$ is called strong Feller operator if $T f \in C(K)$ for every $f \in B_{b}(K)$. A kernel operator $T$ on $C(K)$ is called strong Feller operator if its canonical extension $\tilde{T}$ is a strong Feller operator. 
Let us now consider a bounded, open set $\Omega \subset \mathbb{R}^{d}$ and put $K:=\bar{\Omega}$. In what follows, we will be concerned with operators $T \in \mathscr{L}\left(L^{\infty}(\Omega)\right)$ which take values $C(K)$. It would be tempting to also call such an operator a strong Feller operator, but there are some subtleties in this situation. Let us explain this a little bit.

The concept of 'strong Feller operator' is only useful for kernel operators. Give an operator $T \in \mathscr{L}\left(L^{\infty}(\Omega)\right)$ which takes values in $C(K)$, we can consider the restriction $S:=\left.T\right|_{C(K)}$ of $T$ to $C(K)$. As observed above, $S$ is a kernel operator and thus has a canonical extension $\tilde{S}$ to $\mathscr{L}\left(B_{b}(K)\right)$. Now let $\iota: B_{b}(K) \rightarrow L^{\infty}(\Omega)$ map a bounded measurable function to its equivalence class modulo equality almost everywhere. Then the obvious question is whether $T \circ \iota=\tilde{S}$. Example 5.4 in $[8]$ shows that this need not be the case without further assumptions. The problem is that $T \circ \iota$ need not be a kernel operator. However, using the characterization of kernel operators in 2.4 , we obtain

Lemma 2.6. Let $T \in \mathscr{L}\left(L^{\infty}(\Omega)\right)$ take values in $C(\bar{\Omega})$ and let $\iota: B_{b}(\bar{\Omega}) \rightarrow L^{\infty}(\Omega)$ be as above. Then $T \circ \iota$ is a kernel operator if and only if for every bounded sequence $\left(f_{n}\right) \subset L^{\infty}(\Omega)$ converging almost everywhere to $f$, we have $T f_{n}(x) \rightarrow T f(x)$ for all $x \in \bar{\Omega}$. In this case, $T \circ \iota$ is a strong Feller operator.

We define:

Definition 2.7. An operator $T \in \mathscr{L}\left(L^{\infty}(\Omega)\right)$ is called strong Feller operator if

(a) $T f \in C(\bar{\Omega})$ for every $f \in L^{\infty}(\Omega)$ and

(b) for every bounded sequence $\left(f_{n}\right) \subset L^{\infty}(\Omega)$ converging pointwise almost everywhere to $f$, we have $T f_{n} \rightarrow T f$ pointwise.

\section{Greiner's boundary perturbation revisited.}

An important tool in this article is boundary perturbation of the generator of a holomorphic semigroup, established by Greiner in his seminal article [23]. As a matter of fact, we need some extensions of Greiners results whose proofs follow along the lines of Greiners article with minor modifications. More precisely, we will consider semigroups which are not necessarily strongly continuous. Besides being interesting in its own right, this will allow us to establish under appropriate assumptions the strong Feller property for the perturbed semigroup. Likewise, other modifications allow us to prove compactness, positivity and domination for the perturbed semigroup. In an effort of being self contained and for the convenience of the reader we provide complete proofs.

Throughout this section, we make the following assumption.

Hypothesis 3.1. We are given complex Banach spaces $\left(X,\|\cdot\|_{X}\right),\left(D,\|\cdot\|_{D}\right)$ and $\left(\partial X,\|\cdot\|_{\partial X}\right)$, where $D$ is continuously embedded into $X$. We identify $D$ with its image in $X$ and frequently consider the closure $\bar{D}$ of $D$ in $X$. Moreover, we are given a continuous maximal operator $A: D \rightarrow X$, a continuous boundary operator $B: D \rightarrow \partial X$ and a boundary perturbation $\Phi: \bar{D} \rightarrow \partial X$. We assume that all of these mappings are linear and continuous. Moreover, we assume the following.

(a) The boundary operator $B$ is surjective; 
(b) the boundary perturbation $\Phi$ is compact;

(c) the operator $A_{0}:=\left.A\right|_{\operatorname{ker} B}$ generates a holomorphic semigroup on $X$ and we have $\overline{D\left(A_{0}\right)}=\bar{D}$. We denote by $\omega$ a real number such that any $\lambda \in \mathbb{C}$ with $\operatorname{Re} \lambda>\omega$ belongs to $\rho\left(A_{0}\right)$.

In comparison to Greiner's original work, the main difference in our assumption is that we do not assume the operator $A_{0}$ to be densely defined in $X$. Consequently, the semigroup $T$ generated by $A_{0}$ need not be strongly continuous. However, since $\overline{D\left(A_{0}\right)}=\bar{D}$, it follows from Lemma 2.3 that for every $f \in \bar{D}$ the orbit $t \mapsto T(t) f$ is strongly continuous on $[0, \infty)$ and $T$ restricts to a strongly continuous holomorphic semigroup on $\bar{D}$.

Given the above maps, we define the perturbed operator $A_{\Phi}$ by

$$
D\left(A_{\Phi}\right):=\{u \in D: B u=\Phi u\}, \quad A_{\Phi} u=A u .
$$

We can now formulate our version of Greiner's result.

TheOREM 3.2. Assuming Hypothesis 3.1, the operator $A_{\Phi}$ generates a holomorphic semigroup on $X$ which restricts to a strongly continuous and holomorphic semigroup on $\bar{D}$.

We prepare the proof of Theorem 3.2 with some preliminary results.

Lemma 3.3. Assume that $\lambda \in \rho\left(A_{0}\right)$. Then $D=D\left(A_{0}\right) \oplus \operatorname{ker}(\lambda-A)$.

Proof. If $u \in D\left(A_{0}\right) \cap \operatorname{ker}(\lambda-A)$, then $u \in D\left(A_{0}\right)$ satisfies $A_{0} u=\lambda u$. As $\lambda \in \rho\left(A_{0}\right)$ we must have $u=0$. Now let $u \in D$ be arbitrary. Since $\lambda-A_{0}$ is surjective, we find $u_{0} \in D\left(A_{0}\right)$ with $(\lambda-A) u=\left(\lambda-A_{0}\right) u_{0}$. Consequently $u-u_{0} \in \operatorname{ker}(\lambda-A)$ whence $u=u_{0}+\left(u-u_{0}\right) \in D\left(A_{0}\right)+\operatorname{ker}(\lambda-A)$.

In our framework we can formulate well-posedness of the following boundary value problem (3.1).

Lemma 3.4. Let $\lambda \in \rho\left(A_{0}\right)$. Then for every $h \in \partial X$ the problem

$$
\begin{cases}\lambda u-A u & =0, \\ B u & =h\end{cases}
$$

has a unique solution $u=: S_{\lambda} h$ in $D$. The operator $S_{\lambda}: \partial X \rightarrow D$ is continuous, $B S_{\lambda}=I_{\partial X}$ and $S_{\lambda} B$ is the projection onto $\operatorname{ker}(\lambda-A)$ along $D\left(A_{0}\right)$.

Proof. By Lemma 3.3 the map $B$ defines a continuous bijection between $\operatorname{ker}(\lambda-$ $A)$ and $\partial X$. As a consequence of the open mapping theorem $S_{\lambda}:=\left(\left.B\right|_{\operatorname{ker}(\lambda-A)}\right)^{-1}$ is a continuous linear operator from $\partial X$ to $\operatorname{ker}(\lambda-A)$. Obviously, $u:=S_{\lambda} h$ solves (3.1). If $\tilde{u}$ was another solution, we must have $u-\tilde{u} \in \operatorname{ker} B \cap \operatorname{ker}(\lambda-A)=\{0\}$ by Lemma 3.3. This proves uniqueness. The last assertions are obvious from the definition. 
Lemma 3.5. Let $\lambda \in \rho\left(A_{0}\right)$. Then for $u \in \bar{D}$ one has $u \in D\left(A_{\Phi}\right)$ if and only if $\left(I-S_{\lambda} \Phi\right) u \in D\left(A_{0}\right)$. In this case

$$
\left(\lambda-A_{\Phi}\right) u=\left(\lambda-A_{0}\right)\left(I-S_{\lambda} \Phi\right) u
$$

for every $u \in D\left(A_{\Phi}\right)$. In particular, if $\left(I-S_{\lambda} \Phi\right): \bar{D} \rightarrow \bar{D}$ is invertible, we have $\lambda \in \rho\left(A_{\Phi}\right)$ and

$$
R\left(\lambda, A_{\Phi}\right)=\left(I-S_{\lambda} \Phi\right)^{-1} R\left(\lambda, A_{0}\right) .
$$

Proof. Let us first assume that $u \in D\left(A_{\Phi}\right)$, i.e. $u \in D$ and $B u=\Phi u$. Since $B S_{\lambda}=I_{\partial X}$ by Lemma 3.4, we find $B\left(I-S_{\lambda} \Phi\right) u=B u-B S_{\lambda} \Phi u=B u-\Phi u=0$. Thus $\left(I-S_{\lambda} \Phi\right) u \in \operatorname{ker} B$ and consequently $\left(I-S_{\lambda} \Phi\right) u \in D\left(A_{0}\right)$.

Conversely, if we assume that $u-S_{\lambda} \Phi u \in D\left(A_{0}\right)$, then $u=\left(I-S_{\lambda} \Phi\right) u+S_{\lambda} \Phi u \in D$, as $S_{\lambda}$ takes values in $D$, and $B u=B S_{\lambda} \Phi u=\Phi u$ since $B S_{\lambda}=I_{\partial X}$. Thus $u \in D\left(A_{\Phi}\right)$.

Let us now assume that $u \in D\left(A_{\Phi}\right)$ or, equivalently, that $\left(I-S_{\lambda} \Phi\right) u \in D\left(A_{0}\right)$. Then

$$
\left(\lambda-A_{0}\right)\left(I-S_{\lambda} \Phi\right) u=(\lambda-A) u-(\lambda-A) S_{\lambda} \Phi u=(\lambda-A) u
$$

since $S_{\lambda}$ takes values in $\operatorname{ker}(\lambda-A)$. This implies (3.2).

We now obtain the following criterion to prove that $A_{\Phi}$ generates a holomorphic semigroup.

Proposition 3.6. Assume that there is some $\rho>\omega$ such that for $\lambda \in \mathbb{C}$ with $\operatorname{Re} \lambda>\rho$ the map $I-S_{\lambda} \Phi$ is invertible with

$$
C:=\sup _{\operatorname{Re} \lambda>\rho}\left\|\left(I-S_{\lambda} \Phi\right)^{-1}\right\|_{\mathscr{L}(\bar{D})}<\infty .
$$

Then $A_{\Phi}$ generates a holomorphic semigroup on $X$.

Proof. Set

$$
M:=\sup _{\operatorname{Re} \lambda>\rho}\left\|\lambda R\left(\lambda, A_{0}\right)\right\|<\infty
$$

since $A_{0}$ generates a holomorphic semigroup. As a consequence of Lemma 3.5, for $\operatorname{Re} \lambda>$ $\rho$ we have $\lambda \in \rho\left(A_{\Phi}\right)$ and

$$
\left\|\lambda R\left(\lambda, A_{\Phi}\right)\right\|=\left\|\lambda\left(I-S_{\lambda} \Phi\right)^{-1} R\left(\lambda, A_{0}\right)\right\| \leq\left\|\left(I-S_{\lambda} \Phi\right)^{-1}\right\|\left\|\lambda R\left(\lambda, A_{0}\right)\right\| \leq C M .
$$

By Theorem 2.2, this implies that $A_{\Phi}$ generates a holomorphic semigroup on $X$.

We can now prove the main result of this section.

Proof of TheOrem 3.2. In view of Proposition 3.6, making use of the Neumann series, it suffices to prove that $S_{\lambda} \Phi \rightarrow 0$ in $\mathscr{L}(\bar{D})$ as $\operatorname{Re} \lambda \rightarrow \infty$. Since $\Phi: \bar{D} \rightarrow \partial X$ is compact it suffices to prove that $S_{\lambda} h \rightarrow 0$ as $\operatorname{Re} \lambda \rightarrow \infty$ for every $h \in \partial X$. 
To prove this, let $h \in \partial X$ and fix $\mu \in \rho\left(A_{0}\right)$. We put

$$
u_{\lambda}:=S_{\lambda} h, \quad u_{\mu}:=S_{\mu} h \quad \text { and } \quad u=u_{\lambda}-u_{\mu} .
$$

Note that $u_{\lambda}, u_{\mu}, u \in D$ and that $u \in D\left(A_{0}\right)$. Since $u_{\lambda} \in \operatorname{ker}(\lambda-A)$ and $u_{\mu} \in \operatorname{ker}(\mu-A)$ we have

$$
\left(\lambda-A_{0}\right) u=-(\lambda-A) u_{\mu}=(\mu-\lambda) u_{\mu}
$$

and hence $u=(\mu-\lambda) R\left(\lambda, A_{0}\right) u_{\mu}$. Consequently,

$$
u_{\lambda}=u_{\mu}-\lambda R\left(\lambda, A_{0}\right) u_{\mu}+\mu R\left(\lambda, A_{0}\right) u_{\mu} \rightarrow u_{\mu}-u_{\mu}+0=0
$$

as $\operatorname{Re} \lambda \rightarrow \infty$, since $\lambda R\left(\lambda, A_{0}\right) f \rightarrow f$ for every $f \in \overline{D\left(A_{0}\right)}=\bar{D}$.

We can now establish some additional properties of the operator $A_{\Phi}$ and the semigroup generated by it. We start with compactness.

Corollary 3.7. In the situation of Theorem 3.2, if $A_{0}$ has compact resolvent, then so does $A_{\Phi}$.

Proof. This follows immediately from the identity (3.2) and the ideal property of compact operators.

Next we address positivity of the semigroup. Most often we will be concerned with Banach lattices such as $C(\bar{\Omega})$ or $L^{\infty}(\Omega)$. However, we will occasionally (for example in the following corollaries) also consider closed subspaces of such spaces and therefore need the notion of positivity also in a more general setting. To that end, we assume that our Banach space $X$ is the complexification of a real ordered Banach space $X_{\mathbb{R}}$. This means that in the real Banach space $X_{\mathbb{R}}$ a positive, proper, closed cone $X_{+}$is given, i.e. we have $X_{+}+X_{+} \subset X_{+}, \mathbb{R}_{+} \cdot X_{+} \subset X_{+}$and $X_{+} \cap\left(-X_{+}\right)=\{0\}$. For $u \in X$ we write $u \geq 0$ if $u \in X_{+}$. An operator $S: X \rightarrow X$ is called positive if $S X_{+} \subset X_{+}$, we write $S \geq 0$. Given two operators $S_{1}, S_{2}: X \rightarrow X$, we write $S_{1} \leq S_{2}$ if $S_{2}-S_{1} \geq 0$. A semigroup $T$ on $X$ is called positive if $T(t) \geq 0$ for all $t>0$.

If $Y \subset X$ is a closed subspace of $X$, then $Y_{+}:=Y \cap X_{+}$is a closed, proper cone, such that $Y_{\mathbb{R}}:=Y \cap X_{\mathbb{R}}$ becomes an ordered Banach space. Note that we do not assume that our cone is generating, i.e. we do not necessarily have that $X_{+}-X_{+}=X_{\mathbb{R}}$.

Corollary 3.8. Assume in addition to Hypothesis 3.1 that $X$ is the complexification of a real ordered Banach space and that $A_{0}$ generates a positive semigroup. If there is a $\rho>\omega$ such that for $\lambda \in \mathbb{R}$ with $\lambda>\rho$ the operator $S_{\lambda} \Phi$ is positive, then also the semigroup generated by $A_{\Phi}$ is positive.

Proof. If the semigroup $T$ generated by $A_{0}$ is positive then we have $R\left(\lambda, A_{0}\right) \geq 0$ for $\lambda>\omega$, as the resolvent is given as the Laplace transform of the semigroup. For sufficiently large $\lambda \in \mathbb{R}$ we have $\left\|S_{\lambda} \Phi\right\|<1$ and $S_{\lambda} \Phi$ positive. Thus, by the Neumann series, 


$$
\left(I-S_{\lambda} \Phi\right)^{-1}=\sum_{n=0}^{\infty}\left(S_{\lambda} \Phi\right)^{n}
$$

is a positive operator. It follows from (3.2) that $R\left(\lambda, A_{\Phi}\right)$ is positive for sufficiently large $\lambda$. It follows from the Post-Widder inversion formula [6, Theorem 1.7.7] that the semigroup generated by $A_{\Phi}$ is positive.

Next we want to compare different perturbations of our operator $A$. We can obtain different perturbations by either using different boundary operators $B$ or by using different boundary perturbations $\Phi$.

Corollary 3.9. Let $X, D, \partial X$ and $A$ be as in Hypothesis 3.1 and assume that $X$ and $\partial X$ are complexifications of real ordered Banach spaces. Moreover, assume that we are given maps $B_{1}, B_{2}: D \rightarrow \partial X$ and $\Phi_{1}, \Phi_{2}: \bar{D} \rightarrow \partial X$ such that Hypothesis 3.1 is satisfied for the operators $A, B_{1}, \Phi_{1}$ and the operators $A, B_{2}, \Phi_{2}$. We write $A_{0}^{j}:=\left.A\right|_{\operatorname{ker} B_{j}}$ and $S_{\lambda}^{j}:=\left(\left.B_{j}\right|_{\operatorname{ker}(\lambda-A)}\right)^{-1}$ for $j=1,2$. Finally, we assume that

(a) The semigroup generated by $A_{0}^{j}$ is positive for $j=1,2$;

(b) $0 \leq \Phi_{1} \leq \Phi_{2}$;

(c) For some $\rho>\omega$ and all $\lambda>\rho$ we have $0 \leq S_{\lambda}^{1} \leq S_{\lambda}^{2}$;

(d) If $u \in D$ is positive, then $B_{2} u \leq B_{1} u$.

Then for the semigroups $T_{1}$ generated by $A_{\Phi_{1}}^{1}$ and $T_{2}$ generated by $A_{\Phi_{2}}^{2}$ we have $0 \leq$ $T_{1}(t) \leq T_{2}(t)$ for all $t>0$.

Proof. Let us first note that since the operators $\Phi_{j}$ and $S_{\lambda}^{j}$ are positive for $\lambda>\rho$ and $j=1,2$, it follows from Corollary 3.8 that $T_{1}$ and $T_{2}$ are positive semigroups. It follows from (b) and (c) that

$$
\left(I-S_{\lambda}^{1} \Phi_{1}\right)^{-1}=\sum_{n=0}^{\infty}\left(S_{\lambda}^{1} \Phi_{1}\right)^{n} \leq \sum_{n=0}^{\infty}\left(S_{\lambda}^{2} \Phi_{2}\right)^{n}=\left(I-S_{\lambda}^{2} \Phi_{2}\right)^{-1}
$$

for all $\lambda>\omega$. Now fix $f \geq 0$ and $\lambda>\rho$. We put $u_{j}:=R\left(\lambda, A_{0}^{j}\right) f$. Then $(\lambda-A)\left(u_{1}-u_{2}\right)=$ 0 and $B_{1} u_{1}=B_{2} u_{2}=0$. Using our assumption (d) and the fact that $u_{1} \geq 0$, we see that

$$
B_{2}\left(u_{1}-u_{2}\right)=B_{2} u_{1}-B_{1} u_{1} \leq 0 .
$$

Consequently, as $u_{1}-u_{2}=S_{\lambda}^{2}\left(B_{2}\left(u_{1}-u_{2}\right)\right)$ and $S_{\lambda}^{2}$ is positive $u_{1}-u_{2} \leq 0$. This proves $R\left(\lambda, A_{0}^{1}\right) \leq R\left(\lambda, A_{0}^{2}\right)$. Combining this with the above and Equation (3.2), we find

$$
R\left(\lambda, A_{\Phi_{1}}^{1}\right)=\left(I-S_{\lambda}^{1} \Phi_{1}\right)^{-1} R\left(\lambda, A_{0}^{1}\right) \leq\left(I-S_{\lambda}^{2} \Phi_{2}\right)^{-1} R\left(\lambda, A_{0}^{2}\right)=R\left(\lambda, A_{\Phi_{2}}^{2}\right)
$$

for all sufficiently large $\lambda$. By the Post-Widder inversion formula [6, Theorem 1.7.7] it follows that $T_{1} \leq T_{2}$. 
Our last topic is the strong Feller property for the semigroup generated by the perturbed operator.

Corollary 3.10. Assume in addition to Hypothesis 3.1 that $X=L^{\infty}(\Omega)$ and $\bar{D}=C(\bar{\Omega})$ for some open and bounded $\Omega \subset \mathbb{R}^{d}$. If $A_{0}$ generates a strong Feller semigroup on $X$, then so does $A_{\Phi}$.

Proof. By the proof of [8, Corollary 5.8] it suffices to prove that for sufficiently large $\operatorname{Re} \lambda$ the operator $R\left(\lambda, A_{\Phi}\right)$ is a strong Feller operator. But this follows from (3.2): The hypothesis implies that $R\left(\lambda, A_{0}\right)$ is a strong Feller operator, in particular it maps $L^{\infty}(\Omega)$ to $C(\bar{\Omega})$. Since $U:=\left(I-S_{\lambda} \Phi\right)^{-1}$ is a bounded linear operator on $C(\bar{\Omega})$ also $R\left(\lambda, A_{\Phi}\right)$ maps $L^{\infty}(\Omega)$ to $C(\bar{\Omega})$. Moreover, if $f_{n}$ is a bounded sequence in $L^{\infty}(\Omega)$ converging pointwise almost everywhere to $f$, then $R\left(\lambda, A_{0}\right) f_{n}$ is a bounded sequence which converges pointwise to $R\left(\lambda, A_{0}\right) f$. Since $U$ is bounded on $C(\bar{\Omega})$ we have for $x \in \bar{\Omega}$

$$
\begin{aligned}
R\left(\lambda, A_{\Phi}\right) f_{n}(x) & =\left\langle U R\left(\lambda, A_{0}\right) f_{n}, \delta_{x}\right\rangle=\left\langle R\left(\lambda, A_{0}\right) f_{n}, U^{*} \delta_{x}\right\rangle \\
& \rightarrow\left\langle R\left(\lambda, A_{0}\right) f, U^{*} \delta_{x}\right\rangle=R\left(\lambda, A_{\Phi}\right) f(x),
\end{aligned}
$$

where we have used dominated convergence.

\section{Local Robin boundary conditions.}

In this section we collect some results on elliptic operators with local Robin boundary conditions which we will need in the next section when we establish our results concerning non-local boundary conditions.

Let $\Omega \subset \mathbb{R}^{d}$ be a bounded open set with Lipschitz boundary. As we are talking about positive semigroups, we will consider real-valued spaces $L^{p}(\Omega), C(\bar{\Omega}), C_{b}(\Omega)$ and $B_{b}(\Omega)$ throughout. Only when we are concerned with holomorphic semigroups we need spaces of complex-valued functions, in which case we pass to the complexification of these spaces. Concerning the coefficients of our operator we make the following assumptions.

Hypothesis 4.1. We are given bounded, real-valued, measurable functions $a_{i j}$, $b_{j}, c_{j}, d_{0}$ on $\Omega$ for $i, j=1, \ldots, d$. The diffusion coefficients $a=\left(a_{i j}\right)$ are assumed to be bounded and strictly elliptic, i.e. there is a constant $\eta>0$ such that for all $\xi \in \mathbb{R}^{d}$ and almost all $x \in \Omega$ we have

$$
\sum_{i, j=1}^{d} a_{i j}(x) \xi_{i} \xi_{j} \geq \eta|\xi|^{2}
$$

With these assumptions we define the operator $\mathscr{A}: H^{1}(\Omega) \rightarrow \mathscr{D}(\Omega)^{\prime}$ by

$$
\mathscr{A} u:=-\sum_{i, j=1}^{d} D_{i}\left(a_{i j} D_{j} u\right)-\sum_{j=1}^{d} D_{j}\left(b_{j} u\right)+\sum_{j=1}^{d} c_{j} D_{j} u+d_{0} u .
$$

Here, $H^{1}(\Omega)$ denotes the usual Sobolev space of order one, $\mathscr{D}(\Omega)=C_{c}^{\infty}(\Omega)$ is the space of all test functions and $\mathscr{D}(\Omega)^{\prime}$ is the space of all distributions. We introduce the continuous 
bilinear form $\mathfrak{a}: H^{1}(\Omega) \times H^{1}(\Omega) \rightarrow \mathbb{R}$ given by

$$
\mathfrak{a}[u, v]:=\sum_{i, j=1}^{d} \int_{\Omega} a_{i j} D_{i} u D_{j} v \mathrm{~d} x+\sum_{j=1}^{d} \int_{\Omega} b_{j} u D_{j} v+c_{j}\left(D_{j} u\right) v \mathrm{~d} x+\int_{\Omega} d_{0} u v \mathrm{~d} x
$$

for $u, v \in H^{1}(\Omega)$. Thus $\langle\mathscr{A} u, \varphi\rangle=\mathfrak{a}[u, \varphi]$ for all $u \in H^{1}(\Omega)$ and $\varphi \in \mathscr{D}(\Omega)$.

If $u \in H^{1}(\Omega)$, we say that $\mathscr{A} u \in L^{2}(\Omega)$ if there exists a function $f \in L^{2}(\Omega)$ such that $\langle\mathscr{A} u, \varphi\rangle=[f, \varphi]$ for all $\varphi \in \mathscr{D}(\Omega)$. Here, and in what follows,

$$
[f, g]:=\int_{\Omega} f g \mathrm{~d} x
$$

denotes the scalar product in $L^{2}(\Omega)$. If $\mathscr{A} u \in L^{2}(\Omega)$ the function $f$ above is unique and we identify $\mathscr{A} u$ and $f$.

Next we define the weak conormal derivative by testing against functions in $H^{1}(\Omega)$ rather than functions in $\mathscr{D}(\Omega)$ only.

Definition 4.2. Let $u \in H^{1}(\Omega)$ be such that $\mathscr{A} u \in L^{2}(\Omega)$. For a function $h \in L^{2}(\partial \Omega)$ we say that $h$ is the weak conormal derivative of $u$ and write $\partial_{\nu}^{\mathscr{A}} u:=h$ if the Green formula

$$
\mathfrak{a}[u, v]-[\mathscr{A} u, v]=\int_{\partial \Omega} h v \mathrm{~d} \sigma
$$

holds for all $v \in H^{1}(\Omega)$.

Under our assumptions on the coefficients the weak conormal derivative, if it exists, is unique. It depends on the operator $\mathscr{A}$ only through the coefficients $a=\left(a_{i j}\right)$ and $b_{j}$. Moreover, if the coefficients and the boundary of $\Omega$ are smooth enough the weak conormal derivative coincides with the usual conormal derivative

$$
\partial_{\nu}^{\mathscr{A}} u=\sum_{j=1}^{d}\left(\sum_{i=1}^{d} a_{i j} D_{i} u+\operatorname{tr} b_{j} u\right) \nu_{j}
$$

where $\nu=\left(\nu_{1}, \ldots, \nu_{d}\right)$ is the unit outer normal of $\Omega$. In particular, $\partial_{\nu}^{\mathscr{A}} \mathbb{1}=\sum_{j=1}^{d} \operatorname{tr} b_{j} \nu_{j}$. For a proof of these facts and more information we refer to [1, Section 8.1].

Next we endow our differential operator with Robin boundary conditions, given through a real function $\beta \in L^{\infty}(\partial \Omega)$. For now, we do not (as in Hypothesis 1.1) assume that $\beta \geq 0$, but this assumption will be used later on (in Theorem 4.10) to obtain analyticity of the semigroup via Gaussian estimates.

To define the differential operator with Robin boundary conditions, we employ the theory of bilinear forms, defining $\mathfrak{a}_{\beta}: H^{1}(\Omega) \times H^{1}(\Omega) \rightarrow \mathbb{R}$ by

$$
\mathfrak{a}_{\beta}[u, v]:=\mathfrak{a}[u, v]+\int_{\partial \Omega} \beta u v \mathrm{~d} \sigma .
$$

The associated operator $\mathscr{A}_{\beta}^{2}$ on $L^{2}(\Omega)$ is given by 


$$
\begin{aligned}
D\left(\mathscr{A}_{\beta}^{2}\right) & :=\left\{u \in H^{1}(\Omega): \exists f \in L^{2}(\Omega) \text { with } \mathfrak{a}_{\beta}[u, v]=[f, v] \forall v \in H^{1}(\Omega)\right\}, \\
\mathscr{A}_{\beta}^{2} u & :=f .
\end{aligned}
$$

Testing against test functions we see that $\mathscr{A}_{\beta}^{2} u=\mathscr{A} u$ for all $u \in D\left(\mathscr{A}_{\beta}^{2}\right)$. By the definition of the weak conormal derivative we obtain the following description of the domain:

$$
D\left(\mathscr{A}_{\beta}^{2}\right)=\left\{u \in H^{1}(\Omega): \mathscr{A} u \in L^{2}(\Omega) \text { and } \partial_{\nu}^{\mathscr{A}} u+\beta \operatorname{tr} u=0\right\} .
$$

Thus $\mathscr{A}_{\beta}^{2}$ is the realization of $\mathscr{A}$ with Robin boundary condition. We immediately obtain the following generation result.

Proposition 4.3. Assume Hypothesis 4.1 and let $\beta \in L^{\infty}(\partial \Omega)$. Then the operator $-\mathscr{A}_{\beta}^{2}$ generates a positive, strongly continuous semigroup $T_{\beta}^{2}$ on $L^{2}(\Omega)$.

Proof. Using Lemma 4.7 below and the fact that the trace is a compact operator from $H^{1}(\Omega)$ to $L^{2}(\partial \Omega)$, we see that the form $\mathfrak{a}_{\beta}$ is elliptic, i.e. there are constants $\alpha>0$ and $\omega \geq 0$ such that

$$
\mathfrak{a}_{\beta}[u, u]+\omega\|u\|_{L^{2}(\Omega)}^{2} \geq \alpha\|u\|_{H^{1}(\Omega)} .
$$

Thus, by standard results from the theory of quadratic forms ([31, Section 1.4]) $-\mathscr{A}_{\beta}^{2}$ generates a holomorphic semigroup $T_{\beta}^{2}$. The positivity of $T_{\beta}^{2}$ follows from $[\mathbf{3 1}$, Theorem 2.6] noting that $\mathfrak{a}_{\beta}\left[u^{+}, u^{-}\right]=0$ for all $u \in H^{1}(\Omega)$.

We next investigate when the semigroup $T_{\beta}^{2}$ is sub-Markovian. We will use the following lemma.

Lemma 4.4. Let $g \in L^{2}(\Omega)$ and $h \in L^{2}(\partial \Omega)$ be such that

$$
\int_{\Omega} g v \mathrm{~d} x+\int_{\partial \Omega} h v \mathrm{~d} \sigma \geq 0
$$

for all $0 \leq v \in H^{1}(\Omega)$. Then $g \geq 0$ a.e. on $\Omega$ and $h \geq 0$ a.e. on $\partial \Omega$. Moreover, if in (4.1) identity holds for all $v \in H^{1}(\Omega)$, then $g=0$ a.e. on $\Omega$ and $h=0$ a.e. on $\partial \Omega$.

Proof. By (4.1) we have $\int_{\Omega} g v \mathrm{~d} x \geq 0$ for all $0 \leq v \in C_{c}^{\infty}(\Omega)$. Thus $g \geq 0$ almost everywhere on $\Omega$. Given a function $\varphi \in C(\partial \Omega)$, we find a sequence $v_{n} \in C^{\infty}(\bar{\Omega})$ such that $\left.v_{n}\right|_{\partial \Omega} \rightarrow \varphi$ in $C(\partial \Omega), 0 \leq v_{n} \leq\|\varphi\|_{\infty}$ in $\Omega$ and such that $v_{n}$ is supported in a relatively open set $U_{n} \subset \bar{\Omega}$ with $U_{n} \supset U_{n+1}$ and $\bigcap_{n \in \mathbb{N}} U_{n}=\partial \Omega$. Choosing $v=v_{n}$ in (4.1) and letting $n \rightarrow \infty$, we infer from dominated convergence that $\int_{\partial \Omega} h \varphi \mathrm{d} \sigma \geq 0$. As $\varphi \in C(\partial \Omega)$ was arbitrary, the claim follows.

Proposition 4.5. Assume Hypothesis 4.1 and let $\beta \in L^{\infty}(\partial \Omega)$. We additionally assume that $b_{j} \in W^{1, \infty}(\Omega)$ for $j=1, \ldots, d$.

(a) The semigroup $T_{\beta}^{2}$ is sub-Markovian if and only if 


$$
\begin{gathered}
\sum_{j=1}^{d} D_{j} b_{j} \leq d_{0} \quad \text { almost everywhere on } \Omega \text { and } \\
\sum_{j=1}^{d} \operatorname{tr}\left(b_{j}\right) \nu_{j}+\beta \geq 0 \quad \text { almost everywhere on } \partial \Omega .
\end{gathered}
$$

(b) The semigroup $T_{\beta}^{2}$ is Markovian if and only if

$$
\begin{gathered}
\sum_{j=1}^{d} D_{j} b_{j}=d_{0} \quad \text { almost everywhere on } \Omega \text { and } \\
\sum_{j=1}^{d} \operatorname{tr}\left(b_{j}\right) \nu_{j}+\beta=0 \quad \text { almost everywhere on } \partial \Omega .
\end{gathered}
$$

Proof. (a) The semigroup $T_{\beta}^{2}$ is sub-Markovian if and only if the Beurling-DenyOuhabaz criterion holds, i.e.

$$
\mathfrak{a}_{\beta}\left[u \wedge 1,(u-1)^{+}\right] \geq 0
$$

for all $u \in H^{1}(\Omega)$, see [31, Chapter 2] and [29, Corollary 2.8] or [17] for the case where the form is not necessarily accretive. Recall that for $u \in H^{1}(\Omega)$ the functions $u \wedge 1$ and $(u-1)^{+}$also belong to $H^{1}(\Omega)$ and

$$
D_{j}(u \wedge 1)=\mathbb{1}_{\{u<1\}} D_{j} u \quad \text { and } \quad D_{j}(u-1)^{+}=\mathbb{1}_{\{u>1\}} D_{j} u .
$$

Thus $D_{i}(u \wedge 1) D_{j}(u-1)^{+}=0$ and $(u-1)^{+} D_{j}(u \wedge 1)=0$. We see that

$$
\begin{gathered}
\mathfrak{a}_{\beta}\left[u \wedge 1,(u-1)^{+}\right] \\
=\int_{\Omega} \sum_{j=1}^{d} b_{j} D_{j}(u-1)^{+} \mathrm{d} x+\int_{\{u>1\}} d_{0}(u-1)^{+} \mathrm{d} x+\int_{\partial \Omega} \beta(u-1)^{+} \mathrm{d} \sigma \\
=-\int_{\Omega} \sum_{j=1}^{d}\left(D_{j} b_{j}\right)(u-1)^{+} \mathrm{d} x+\int_{\partial \Omega} \sum_{j=1}^{d} b_{j} \nu_{j}(u-1)^{+} \mathrm{d} \sigma \\
\quad+\int_{\Omega} d_{0}(u-1)^{+} \mathrm{d} x+\int_{\partial \Omega} \beta(u-1)^{+} \mathrm{d} \sigma .
\end{gathered}
$$

The latter is positive if (4.2) and (4.3) hold whence $T_{\beta}^{2}$ is sub-Markovian in this case. This shows sufficiency of these two conditions.

Conversely, if the semigroup $T_{\beta}^{2}$ is sub-Markovian, the Beurling-Deny-Ouhabaz criterion yields

$$
\int_{\Omega}\left(d_{0}-\sum_{j=1}^{d} D_{j} b_{j}\right)(u-1)^{+} \mathrm{d} x+\int_{\partial \Omega}\left(\sum_{j=1}^{d} b_{j} \nu_{j}+\beta\right)(u-1)^{+} \mathrm{d} \sigma \geq 0
$$


for all $u \in H^{1}(\Omega)$. Choosing $u=\mathbb{1}+v$ with $0 \leq v \in H^{1}(\Omega)$, Lemma 4.4 shows that (4.2) and (4.3) are valid.

(b) A Markovian semigroup is in particular sub-Markovian whence the inequalities (4.2) and (4.3) are satisfied. If $T_{\beta}^{2}$ is sub-Markovian, then it is Markovian if and only if $\mathbb{1} \in \operatorname{ker}\left(-\mathscr{A}_{\beta}^{2}\right)$. Note that

$$
-\mathscr{A} \mathbb{1}=\sum_{j=1}^{d} D_{j} b_{j}-d_{0} .
$$

Thus (4.4) is necessary for $T_{\beta}^{2}$ to be Markovian. If (4.4) holds, then for $v \in H^{1}(\Omega)$ we have

$$
\mathfrak{a}[\mathbb{1}, v]-[\mathscr{A} \mathbb{1}, v]=\sum_{j=1}^{d} \int_{\Omega}\left(b_{j} D_{j} v+d_{0} v\right) \mathrm{d} x=\sum_{j=1}^{d} \int_{\partial \Omega} b_{j} \nu_{j} v \mathrm{~d} \sigma
$$

where we used an integration by parts. Thus saying $\partial_{\nu}^{\mathscr{A}} \mathbb{1}+\beta=0$, i.e. $\mathbb{1} \in D\left(-\mathscr{A}_{\beta}^{2}\right)$, is equivalent to

$$
\sum_{j=1}^{d} \int_{\partial \Omega} b_{j} \nu_{j} v \mathrm{~d} \sigma=-\int_{\partial \Omega} \beta v \mathrm{~d} \sigma
$$

for all $v \in H^{1}(\Omega)$ and hence to (4.5).

In order to apply the abstract results of Section 3, we need some results about the following elliptic problem, which are also used implicitly in the proof of Theorem 4.10.

$$
\left\{\begin{array}{c}
\lambda u+\mathscr{A} u=f \text { on } \Omega \\
\partial_{\nu}^{\mathscr{A}} u+\beta u=h \text { on } \partial \Omega .
\end{array}\right.
$$

Obviously, $\mathfrak{a}_{\beta}$ defines a continuous sesquilinear mapping on $H^{1}(\Omega)$. By [15, Corollary 2.5] it is also elliptic, i.e. there are some $\omega, \alpha>0$ such that $\mathfrak{a}_{\beta}[u, u]+\omega\|u\|_{L^{2}(\Omega)}^{2} \geq \alpha\|u\|_{H^{1}(\Omega)}^{2}$. With this information at hand, one can prove existence and uniqueness of solutions to (4.6) by means of the Lax-Milgram Theorem. Indeed, considering the continuous functional $F$ on $H^{1}(\Omega)$, given by $F(v)=\int_{\Omega} f v \mathrm{~d} x+\int_{\partial \Omega} h v \mathrm{~d} \sigma$, it follows from the LaxMilgram Theorem that for $\lambda>\omega$ there is a unique $u \in H^{1}(\Omega)$ such that

$$
\mathfrak{a}_{\beta}[u, v]+\lambda[u, v]=F(v)
$$

for all $v \in H^{1}(\Omega)$. From [30, Theorem 3.14(iv)] we obtain the following result concerning regularity of the solution.

Proposition 4.6. Assume Hypothesis 4.1, fix $q>d$ and $\lambda>\omega$. Then there exist constants $\gamma>0$ and $C>0$ such that whenever $f \in L^{q / 2}(\Omega)$ and $h \in L^{q-1}(\partial \Omega)$ the unique solution $u$ of (4.6) belongs to $C^{\gamma}(\bar{\Omega})$ and we have

$$
\|u\|_{C^{\gamma}(\bar{\Omega})} \leq C\left(\|f\|_{L^{q / 2}(\Omega)}+\|h\|_{L^{q-1}(\partial \Omega)}\right) .
$$


The following lemma is easy to prove, see e.g. [9, Lemma 2.3].

Lemma 4.7. Let $X_{1}, X_{2}, X_{3}$ be Banach spaces such that $X_{1}$ is reflexive. Let $T$ : $X_{1} \rightarrow X_{3}$ be compact, $S: X_{1} \rightarrow X_{2}$ be injective. Then, given $\varepsilon>0$ there exists a constant $c>0$ such that

$$
\|T x\|_{X_{3}} \leq \varepsilon\|x\|_{X_{1}}+c\|S x\|_{X_{2}}
$$

for all $x \in X_{1}$.

We use this lemma to prove the following domination result.

Proposition 4.8. Assume Hypothesis 4.1 and let $\beta_{1}, \beta_{2} \in L^{\infty}(\partial \Omega)$ be such that $\beta_{1} \leq \beta_{2}$. There exists $\omega$ so that both $\mathfrak{a}_{\beta_{1}}+\omega$ and $\mathfrak{a}_{\beta_{2}}+\omega$ are coercive and such that for $\lambda>\omega$ the following holds. Let $0 \leq f \in L^{2}(\Omega), 0 \leq h \in L^{2}(\partial \Omega)$. For $j=1,2$, let $u_{j} \in H^{1}(\Omega)$ be the unique solution of

$$
\left\{\begin{aligned}
\lambda u+\mathscr{A} u & =f \text { on } \Omega \\
\partial_{\nu}^{\mathscr{A}} u+\beta_{j} u & =h \text { on } \partial \Omega .
\end{aligned}\right.
$$

Then $0 \leq u_{2} \leq u_{1}$.

ProOF. We first show positivity for weak solutions $u$ of (4.6). To that end consider $f \leq 0$ and $h \leq 0$ for now. If $u$ solves (4.6) we have

$$
\lambda[u, v]+\mathfrak{a}_{\beta}[u, v]=[f, v]+\int_{\partial \Omega} h v \mathrm{~d} \sigma
$$

for all $v \in H^{1}(\Omega)$. Setting $v:=u^{+}$and noting that $\mathfrak{a}_{\beta}\left[u, u^{+}\right]=\mathfrak{a}_{\beta}\left[u^{+}, u^{+}\right]$by the locality of $\mathfrak{a}_{\beta}$, we find

$$
\lambda\left[u^{+}, u^{+}\right]+\mathfrak{a}_{\beta}\left[u^{+}, u^{+}\right]=\left[f, u^{+}\right]+\int_{\partial \Omega} h u^{+} \mathrm{d} \sigma \leq 0 .
$$

As $\mathfrak{a}_{\beta}+\omega$ is coercive we have that $\mathfrak{a}_{\beta}\left[u^{+}, u^{+}\right]+\omega\left\|u^{+}\right\|_{L^{2}(\Omega)}^{2} \geq \alpha\left\|u^{+}\right\|_{H^{1}(\Omega)}^{2}$ for some $\alpha>0$. Together with $\lambda>\omega$ it follows that $\left\|u^{+}\right\|_{H^{1}(\Omega)} \leq 0$, whence $u \leq 0$.

We can prove the domination similarly. This time we fix $f \geq 0$ and $h \geq 0$. The solution $u_{j}(j=1,2)$ satisfies the equation

$$
\lambda\left[u_{j}, v\right]+\mathfrak{a}_{\beta_{j}}\left[u_{j}, v\right]=[f, v]+\int_{\partial \Omega} h v \mathrm{~d} \sigma
$$

for all $v \in H^{1}(\Omega)$. Subtracting these equations we find for a positive $v$ that

$$
\lambda\left[u_{2}-u_{1}, v\right]+\mathfrak{a}\left[u_{2}-u_{1}, v\right]=\int_{\partial \Omega}\left(\beta_{1} u_{1}-\beta_{2} u_{2}\right) v \mathrm{~d} \sigma \leq \int_{\partial \Omega} \beta_{2}\left(u_{1}-u_{2}\right) v \mathrm{~d} \sigma,
$$

since $u_{1} \geq 0$ by the above. Testing against $v:=\left(u_{2}-u_{1}\right)^{+}$, we find 


$$
\begin{aligned}
& \lambda\left[\left(u_{2}-u_{1}\right)^{+},\left(u_{2}-u_{1}\right)^{+}\right]+\mathfrak{a}\left[\left(u_{2}-u_{1}\right)^{+},\left(u_{2}-u_{1}\right)^{+}\right] \\
\leq & -\int_{\partial \Omega} \beta_{2}\left(\left(u_{2}-u_{1}\right)^{+}\right)^{2} \mathrm{~d} x \leq\left\|\beta_{2}\right\|_{L^{\infty}(\partial \Omega)} \int_{\partial \Omega}\left(\left(u_{2}-u_{1}\right)^{+}\right)^{2} \mathrm{~d} \sigma .
\end{aligned}
$$

Applying Lemma 4.7 with $X_{1}=H^{1}(\Omega), X_{2}=L^{2}(\Omega)$ and $X_{3}=L^{2}(\partial \Omega)$ where $T$ : $H^{1}(\Omega) \rightarrow L^{2}(\partial \Omega)$ is the trace operator (which is compact) and $S: H^{1}(\Omega) \rightarrow L^{2}(\Omega)$ is the natural embedding, given $\varepsilon>0$ we find a constant $c>0$ such that

$$
\begin{gathered}
\left\|\beta_{2}\right\|_{L^{\infty}(\partial \Omega)} \int_{\partial \Omega}\left(\left(u_{2}-u_{1}\right)^{+}\right)^{2} \mathrm{~d} \sigma \leq \varepsilon\left\|\left(u_{2}-u_{1}\right)^{+}\right\|_{H^{1}(\Omega)}^{2}+c\left\|\left(u_{2}-u_{1}\right)^{+}\right\|_{L^{2}(\Omega)}^{2} \\
=\varepsilon \int_{\Omega}\left|\nabla\left(u_{2}-u_{1}\right)^{+}\right|^{2} \mathrm{~d} x+(c+\varepsilon) \int_{\Omega}\left(\left(u_{2}-u_{1}\right)^{+}\right)^{2} \mathrm{~d} x .
\end{gathered}
$$

Using the ellipticity of $\mathfrak{a}$ we deduce that, for a suitable constant $\alpha>0$, we have

$$
\begin{aligned}
& (\lambda+\alpha-\omega)\left\|\left(u_{2}-u_{1}\right)^{+}\right\|_{L^{2}(\Omega)}^{2}+\alpha \int_{\Omega}\left|\nabla\left(u_{2}-u_{1}\right)^{+}\right|^{2} \mathrm{~d} x \\
\leq & \varepsilon \int_{\Omega}\left|\nabla\left(u_{2}-u_{1}\right)^{+}\right|^{2} \mathrm{~d} x+(c+\varepsilon)\left\|\left(u_{2}-u_{1}\right)^{+}\right\|_{L^{2}(\Omega)}^{2} .
\end{aligned}
$$

Choosing $\varepsilon=\alpha / 2$ and $\lambda_{0}>\omega+c+\varepsilon+1$, it follows that for $\lambda>\lambda_{0}$ we have $\left(u_{2}-u_{1}\right)^{+}=0$, i.e. $u_{2} \leq u_{1}$.

Proposition 4.8 yields in particular the following monotonicity property.

Corollary 4.9. Assume Hypothesis 4.1 and let $\beta_{1}, \beta_{2} \in L^{\infty}(\Omega)$ be such that $\beta_{1} \leq \beta_{2}$. Then $0 \leq T_{\beta_{2}}^{2}(t) \leq T_{\beta_{1}}^{2}(t)$ for all $t \geq 0$.

Proof. Proposition 4.8 shows that for large $\lambda$ we have $0 \leq\left(\lambda+\mathscr{A}_{\beta_{2}}^{2}\right)^{-1} \leq(\lambda+$ $\left.\mathscr{A}_{\beta_{1}}^{2}\right)^{-1}$. This implies the claim in view of Euler's formula.

For our next result, we assume again that $0 \leq \beta$ as in Hypothesis 1.1. Under this assumption, we will show that the semigroup $T_{\beta}^{2}$ on $L^{2}(\Omega)$ always leaves the space $L^{\infty}(\Omega)$ invariant, even if $T_{\beta}^{2}$ is not sub-Markovian. This follows from Gaussian estimates for the semigroup $T_{\beta}^{2}$ which can be proved under the assumption that $0 \leq \beta$. It seems to be unknown whether this is necessary for the Gaussian estimates. As a second consequence of the Gaussian estimates, we see that the restriction $T_{\beta}$ of $T_{\beta}^{2}$ to $L^{\infty}(\Omega)$ is a holomorphic semigroup, by which we mean that the $\mathbb{C}$-linear extension of $\left.T_{\beta}^{2}\right|_{L^{\infty}(\Omega)}$ to the complexification $L^{\infty}(\Omega ; \mathbb{C})$ of $L^{\infty}(\Omega)$ is holomorphic.

Of course the generator of $T_{\beta}$ is the part $A_{\beta}$ of $-\mathscr{A}_{\beta}^{2}$ in $L^{\infty}(\Omega)$, i.e.

$$
\begin{aligned}
D\left(A_{\beta}\right) & =\left\{u \in H^{1}(\Omega) \cap L^{\infty}(\Omega): \mathscr{A} u \in L^{\infty}(\Omega), \partial_{\nu}^{\mathscr{A}} u+\beta u=0\right\}, \\
A_{\beta} u & =-\mathscr{A} u
\end{aligned}
$$

We will also see that the semigroup $T_{\beta}^{2}$ has leaves the space $C(\bar{\Omega})$ invariant and restrincts to a strongly continuous semigroup on that space. Naturally, the generator of $T_{\beta}^{C}$ is the part $A_{\beta}^{C}$ of $-\mathscr{A}_{\beta}^{2}$ in $C(\bar{\Omega})$, i.e. 


$$
\begin{aligned}
D\left(A_{\beta}^{C}\right) & =\left\{u \in H^{1}(\Omega) \cap C(\bar{\Omega}): \mathscr{A} u \in C(\bar{\Omega}), \partial_{\nu}^{\mathscr{A}} u+\beta \operatorname{tr} u=0\right\}, \\
A_{\beta}^{C} u & =-\mathscr{A} u .
\end{aligned}
$$

As a consequence of the strong continuity of $T_{\beta}^{C}$ we find that $D\left(A_{\beta}^{C}\right)$ is dense in $C(\bar{\Omega})$.

TheOREM 4.10. Assume Hypothesis 4.1 and let $0 \leq \beta \in L^{\infty}(\partial \Omega)$. Then $T_{\beta}^{2}$ leaves the space $L^{\infty}(\Omega)$ invariant. Its restriction $T_{\beta}$ to $L^{\infty}(\Omega)$ is a holomorphic semigroup on $L^{\infty}(\Omega)$. Each operator $T_{\beta}(t), t>0$, is compact and enjoys the strong Feller property. In particular, $C(\bar{\Omega})$ is invariant under $T_{\beta}$. The restriction $T_{\beta}^{C}$ of $T_{\beta}^{2}$ to $C(\bar{\Omega})$ is a strongly continuous and holomorphic semigroup.

Proof. It was proved in [14, Corollary 6.1] (see also [10, Theorem 4.9]) that the semigroup $T_{\beta}^{2}$ has Gaussian estimates so that $T_{\beta}^{2}$ extrapolates to a consistent family of semigroups $T_{\beta}^{q}$ on $L^{q}(\Omega)$ for $q \in[1, \infty]$. In particular, $T_{\beta}^{2}$ leaves the space $L^{\infty}(\Omega)$ invariant and restricts to a semigroup $T_{\beta}$ on this space. By [10, Theorem 5.3] the semigroup $T_{\beta}$ is holomorphic on $L^{\infty}(\Omega)$. Moreover, by the proof of [30, Theorem 4.3] $T_{\beta}(t) L^{\infty}(\Omega) \subset C(\bar{\Omega})$ for all $t>0$. It was also seen in that theorem that $T_{\beta}(t)$ is compact for all $t>0$. We now show that $T_{\beta}(t)$ is strongly Feller for $t>0$. Since $T_{\beta}^{2}$ is ultracontractive by $[\mathbf{3}, 7.3$ Criterion $(\mathrm{v})]$ it follows that $T_{\beta}^{2}(t) L^{q}(\Omega) \subset L^{\infty}(\Omega)$ and hence $T_{\beta}^{2}(t) L^{q}(\Omega) \subset T_{\beta}^{2}(t / 2) L^{\infty}(\Omega) \subset C(\bar{\Omega})$ for some $q \in(2, \infty)$. By the closed graph theorem, $T_{\beta}^{2}(t)$ is a bounded operator from $L^{q}(\Omega)$ to $C(\bar{\Omega})$. Now the strong Feller property, as defined in Definition 2.7, follows from the dominated convergence theorem. It follows from [30, Theorem 4.3] that the restriction of the semigroup to $C(\bar{\Omega})$ is strongly continuous.

\section{Non-local boundary conditions.}

We are now prepared to prove the main results of this article. We begin by setting up the framework in which we apply Greiner's boundary perturbation. In contrast to the last section, in this section only consider complex Banach spaces in order to handle (possibly) complex valued functions $\mu: \partial \Omega \rightarrow \mathscr{M}(\bar{\Omega})$.

We assume throughout Hypotheses 1.1 and 4.1. In particular, we assume throughout that $0 \leq \beta \in L^{\infty}(\partial \Omega)$. We then define

$$
D:=\left\{u \in C(\bar{\Omega}) \cap H^{1}(\Omega): \mathscr{A} u \in L^{\infty}(\Omega), \partial_{\nu}^{\mathscr{A}} u \in L^{p}(\partial \Omega)\right\},
$$

where $p>d-1$ is as in Hypothesis 1.1(b). Endowed with the norm

$$
\|u\|_{D}:=\|u\|_{C(\bar{\Omega})}+\|u\|_{H^{1}(\Omega)}+\|\mathscr{A} u\|_{L^{\infty}(\Omega)}+\left\|\partial_{\nu}^{\mathscr{A}} u\right\|_{L^{p}(\partial \Omega)}
$$

$D$ is a Banach space which is continuously embedded into $X=L^{\infty}(\Omega)$. Since $D\left(A_{\beta}^{C}\right) \subset$ $D$, it follows from Theorem 4.10 that $D$ is dense in $C(\bar{\Omega})$. We define our maximal operator $A: D \rightarrow X$ by $A u:=-\mathscr{A} u$ which is linear and continuous. We set $\partial X:=L^{p}(\partial \Omega)$ and consider the boundary operator $B: D \rightarrow \partial \Omega$ defined via $B u=\partial_{\nu}^{\mathscr{A}} u+\beta u$ where $\beta$ is as in Hypothesis 1.1. Finally, given $\mu$ as in Hypothesis 1.1, the function $\Phi: \bar{D} \rightarrow \partial X$ is given by 


$$
(\Phi u)(z):=\int_{\bar{\Omega}} u(x) \mu(z)(\mathrm{d} x) .
$$

Making use of the results of Section 3 we can now prove our main generation result for the operator $A_{\beta, \mu}$, defined by

$$
\begin{aligned}
D\left(A_{\beta, \mu}\right) & =\left\{u \in C(\bar{\Omega}) \cap H^{1}(\Omega): \mathscr{A} u \in L^{\infty}(\Omega), \partial_{\nu}^{\mathscr{A}} u+\beta u=\langle u, \mu(\cdot)\rangle\right\}, \\
A_{\beta, \mu} & =-\mathscr{A} u .
\end{aligned}
$$

The following result contains Theorem 1.3 from the introduction as a special case.

TheOrem 5.1. Assume Hypotheses 1.1 and 4.1. Then the operator $A_{\beta, \mu}$ generates a holomorphic semigroup $T_{\beta, \mu}$ on $L^{\infty}(\Omega)$ which satisfies the strong Feller property. In particular, it leaves the space $C(\bar{\Omega})$ invariant. Its restriction to this space is a strongly continuous and holomorphic semigroup whose generator is $A_{\beta, \mu}^{C}$, the part of $A_{\beta, \mu}$ in $C(\bar{\Omega})$.

Proof. Noting that the operator $A_{\beta, \mu}$ is exactly the perturbed operator $A_{\Phi}$, where $A$ and $\Phi$ are as defined above, the claim follows immediately from Theorem 3.2 and Corollary 3.10 once we verified that the maps $A, B$ and $\Phi$ satisfy Hypothesis 3.1 .

(a) The operator $B: D \rightarrow \partial X$ is surjective.

Pick $\omega$ such that $\mathfrak{a}+\omega$ is coercive and fix $\lambda>\omega$. Given $h \in \partial X=L^{p}(\partial \Omega)$, it follows from Proposition 4.6 that the unique solution $u \in H^{1}(\Omega)$ of the problem

$$
\left\{\begin{array}{l}
\lambda u+\mathscr{A} u=0 \\
\partial_{\nu}^{\mathscr{A}} u+\beta u=h
\end{array}\right.
$$

belongs to $C(\bar{\Omega})$. Moreover, $\mathscr{A} u=-\lambda u \in C(\bar{\Omega}) \subset L^{\infty}(\Omega)$. Thus, $u \in D$ and $B u=h$, proving that $B$ is surjective.

(b) The boundary map $\Phi$ is compact.

Let $\left(u_{n}\right)_{n \in \mathbb{N}}$ be a bounded sequence in $C(\bar{\Omega})$, say $\left\|u_{n}\right\|_{C(\bar{\Omega})} \leq M$ for all $n \in \mathbb{N}$. Since $\mu(z) \ll \tau$ by Hypothesis 1.1(c), for every $z \in \partial \Omega$ we find a Radon-Nikodym density $\varphi_{z} \in L^{1}(\bar{\Omega}, \tau)$ of $\mu(z)$ with respect to $\tau$, i.e. we have

$$
\int_{\bar{\Omega}} f(x) \mu(z)(\mathrm{d} x)=\int_{\bar{\Omega}} f \varphi_{z} \mathrm{~d} \tau
$$

for all $f \in C(\bar{\Omega})$. In particular, $\left(\Phi u_{n}\right)(z)=\left\langle u_{n}, \varphi_{z}\right\rangle_{L^{\infty}(\tau), L^{1}(\tau)}$. Since the sequence $u_{n}$ is bounded in $L^{\infty}(\tau)$ and $L^{1}(\tau)$ is separable, it follows from the Banach-Alaoglu theorem that we find a weak ${ }^{*}$-convergent subsequence, say $u_{n_{k}} \rightarrow^{*} u$ for some $u \in L^{\infty}(\tau)$. In particular,

$$
\left(\Phi u_{n_{k}}\right)(z)=\int_{\bar{\Omega}} u_{n_{k}} \varphi_{z} \mathrm{~d} \tau \rightarrow \int_{\bar{\Omega}} u \varphi_{z} \mathrm{~d} \tau
$$

for all $z \in \partial \Omega$, i.e. $\Phi u_{n}$ has a subsequence which converges pointwise. Note that we have

$$
\left|\left(\Phi u_{n}\right)(z)\right| \leq M\|\mu(z)\| .
$$


As a consequence of Hypothesis 1.1(b) the functions $\Phi u_{n}$ have a $p$-integrable majorant and it follows from the dominated convergence theorem that $\Phi u_{n}$ has a subsequence which converges in $L^{p}(\partial \Omega)$.

(c) The operator $A_{0}$ is exactly the part of $-\mathscr{A}_{\beta}^{2}$ in $L^{\infty}(\Omega)$. It follows from Theorem 4.10 that $A_{0}$ generates an holomorphic semigroup on $X=L^{\infty}(\Omega)$ which enjoys the strong Feller property and whose domain is dense in $C(\bar{\Omega})$.

We next prove some additional properties of the semigroup $T_{\beta, \mu}$ making use of the corollaries to Theorem 3.2.

Proposition 5.2. Assume Hypotheses 1.1 and 4.1 and let $T_{\beta, \mu}$ be the semigroup generated by $A_{\beta, \mu}$ according to Theorem 5.1.

(a) $T_{\beta, \mu}$ is compact.

(b) If $\mu(z)$ is a positive measure for almost every $z \in \partial \Omega$, then the semigroup $T_{\beta, \mu}$ is positive.

Proof. (a) Follows immediately from Corollary 3.7, noting that the semigroup generated by $A_{0}$ is compact as a consequence of Theorem 4.10.

(b) By Theorem 4.10, the semigroup generated by $A_{0}$ is positive. If $\mu(z)$ is positive for almost every $z \in \partial \Omega$, then the map $\Phi$ is positive. Note that for the solution map $S_{\lambda}$ the function $S_{\lambda} h$ is the unique solution of the boundary value problem

$$
\left\{\begin{array}{l}
\lambda u+\mathscr{A} u=0 \\
\partial_{\nu}^{\mathscr{A}} u+\beta u=h .
\end{array}\right.
$$

Thus, by Proposition $4.8, S_{\lambda}$ is positive for $\lambda>\omega$. Altogether $S_{\lambda} \Phi$ is positive and it follows from Corollary 3.8 that $T_{\beta, \mu}$ is positive.

Next we characterize when $T_{\beta, \mu}$ is Markovian.

Proposition 5.3. Assume in addition to Hypotheses 1.1 and 4.1 that $\mu(z)$ is a positive measure for almost every $z \in \partial \Omega$. The following are equivalent.

(i) The semigroup $T_{\beta, \mu}$ is Markovian.

(ii) We have

$$
\begin{gathered}
\sum_{j=1}^{d} D_{j} b_{j}=d_{0} \quad \text { almost everywhere on } \Omega \text { and } \\
\mu(z)(\bar{\Omega})=\beta(z)+\sum_{j=1}^{d} \nu_{j}(z) b_{j}(z) \quad \text { for almost all } z \in \partial \Omega .
\end{gathered}
$$

Proof. Since $T_{\beta, \mu}$ is positive, (i) is equivalent to $\mathbb{1} \in \operatorname{ker} A_{\beta, \mu}$. Observe that $-\mathscr{A} \mathbb{1}=\sum_{j=1}^{d} D_{j} b_{j}-d_{0}$. Thus $-\mathscr{A} \mathbb{1}=0$ if and only if (5.1) holds. In that case, integration by parts yields for $v \in H^{1}(\Omega)$ that 


$$
\mathfrak{a}[\mathbb{1}, v]-[\mathscr{A} \mathbb{1}, v]=\sum_{j=1}^{d} \int_{\Omega} b_{j} D_{j} v+d_{0} v \mathrm{~d} x=\sum_{j=1}^{d} \int_{\partial \Omega} b_{j} \nu_{j} v \mathrm{~d} \sigma .
$$

Thus $\mathbb{1} \in D\left(A_{\beta, \mu}\right)$ if and only if

$$
\sum_{j=1}^{d} \int_{\partial \Omega} b_{j}(z) \nu_{j}(z) v(z) \mathrm{d} \sigma(z)=\int_{\partial \Omega}(-\beta(z)+\langle\mu(z), \mathbb{1}\rangle) v(z) \mathrm{d} \sigma
$$

for all $v \in H^{1}(\Omega)$. This is equivalent to $(5.2)$.

If we merely have inequalities in (5.1) and (5.2), then the semigroup is sub-Markovian as we show next. In the proof, we use the following monotonicity result.

Proposition 5.4. Assume Hypothesis 4.1 and let $\beta_{1}, \beta_{2} \in L^{\infty}(\partial \Omega)$ with $\beta_{2} \leq \beta_{1}$. Moreover, let functions $\mu_{1}, \mu_{2}: \partial \Omega \rightarrow \mathscr{M}(\bar{\Omega})$ be given such that $0 \leq \mu_{1}(z) \leq \mu_{2}(z)$ for almost all $z \in \partial \Omega$ and such that $\mu_{1}, \mu_{2}$ satisfy Hypothesis 1.1 with the same $p$. Then

$$
0 \leq T_{\beta_{1}, \mu_{1}}(t) \leq T_{\beta_{2}, \mu_{2}}(t)
$$

for all $t \geq 0$.

Proof. The semigroups $T_{\beta_{1}, \mu_{1}}$ and $T_{\beta_{2}, \mu_{2}}$ are obtained from the same maximal operator $A$ but using different boundary perturbations $\Phi_{j}: u \mapsto\left\langle\mu_{j}(\cdot), u\right\rangle$ and boundary operators $B_{j}: u \mapsto \partial_{\nu}^{\mathscr{A}} u+\beta_{j} u$. We clearly have $B_{2} u \leq B_{1} u$ and $0 \leq \Phi_{1} u \leq \Phi_{2} u$ for $u \geq 0$. Moreover, if we write $S_{\lambda}^{j}:=\left(\left.B_{j}\right|_{\operatorname{ker}(\lambda-A)}\right)^{-1}$, then we have $S_{\lambda}^{1} \leq S_{\lambda}^{2}$ by Proposition 4.8. Thus Corollary 3.9 yields the claim.

Proposition 5.5. Assume in addition to Hypotheses 1.1 and 4.1 that $\mu(z)$ is positive for almost all $z \in \partial \Omega$ and that $b_{j} \in W^{1, \infty}(\Omega)$ for $j=1, \ldots, d$. If

$$
\begin{gathered}
\sum_{j=1}^{d} D_{j} b_{j} \leq d_{0} \quad \text { almost everywhere on } \Omega \text { and } \\
\mu(z)(\bar{\Omega}) \leq \beta(z)+\sum_{j=1}^{d} \operatorname{tr}\left(b_{j}\right)(z) \nu_{j}(z) \quad \text { for almost all } z \in \partial \Omega
\end{gathered}
$$

then the semigroup $T_{\beta, \mu}$ is sub-Markovian.

Proof. Assume at first that $\sum_{j=1}^{d} D_{j} b_{j}=d_{0}$. Let us define $\beta_{0}(z):=\mu(z)(\bar{\Omega})-$ $\sum_{j=1}^{d} \operatorname{tr} b_{j}(z) \nu_{j}(z)$. By Proposition 5.3 the semigroup $T_{\beta_{0}, \mu}$ is Markovian. As a consequence of Proposition 5.4 we have $0 \leq T_{\beta, \mu}(t) \leq T_{\beta_{0}, \mu}(t)$ for all $t>0$ which clearly implies that $T_{\beta, \mu}$ is sub-Markovian. That $T_{\beta, \mu}$ is still sub-Markovian when $\sum_{j=1}^{d} D_{j} b_{j} \leq d_{0}$ follows from a standard perturbation result:

Denote by $\tilde{A}_{\beta, \mu}$ the operator where $d_{0}$ is replaced by $\tilde{d}_{0}:=\sum_{j=1}^{d} D_{j} b_{j}$. Then the semigroup $\tilde{T}_{\beta, \mu}$ generated by $\tilde{A}_{\beta, \mu}$ is sub-Markovian by what has been proved so far. Note that $A_{\beta, \mu}+\left(d_{0}-\tilde{d}_{0}\right)=\tilde{A}_{\beta, \mu}$, so that $\tilde{A}_{\beta, \mu}$ is a bounded and positive perturbation 
of $A_{\beta, \mu}$. Using a perturbation result for resolvent positive operators [6, Proposition 3.11.12] we find that $R\left(\lambda, A_{\beta, \mu}\right) \leq R\left(\lambda, \tilde{A}_{\beta, \mu}\right)$ for large enough $\lambda$ and the domination of the semigroups follows from the Post-Widder inversion formula [6, Theorem 1.7.7]. Alternatively, the domination property can be inferred from the Dyson-Phillips formula for the perturbed semigroup, see [26, Example 3.4] for a version which covers our setting.

As a further consequence of Proposition 5.4 we have

$$
0 \leq T_{\beta, 0}(t) \leq T_{\beta, \mu}(t)
$$

for all $t>0$ in the case where $\mu(z)$ is a positive measure for almost every $z \in \partial \Omega$. We note that for $\mu \equiv 0$ we have $T_{\beta, 0}(t)=T_{\beta}(t)$, where $T_{\beta}$ is the semigroup on $L^{\infty}(\Omega)$, defined in Section 4 for local Robin boundary conditions. It thus follows from Proposition 4.5 that condition (5.3) is necessary for $T_{\beta, \mu}$ to be sub-Markovian. It seems not so easy to show that also condition (5.4) is necessary for this. Also concerning the positivity of the semigroup $T_{\beta, \mu}$ it seems unclear if the condition that $\mu(z)$ is a positive measure for almost every $z \in \partial \Omega$ is necessary. However, in Section 8 we will give a proof of necessity in the special case where every measure $\mu(z)$ is absolutely continuous with respect to the Lebesgue measure.

\section{Asymptotic behavior.}

Throughout this section we assume Hypotheses 1.1 and 4.1 so that $T_{\beta, \mu}$ is a semigroup on $L^{\infty}(\Omega)$. It is our aim to describe its asymptotic behavior as $t \rightarrow \infty$. Since $T_{\beta, \mu}(t) L^{\infty}(\Omega) \subset C(\bar{\Omega})$ for all $t>0$ it suffices to study $T_{\beta, \mu}^{C}$, the restriction to $C(\bar{\Omega})$, which is a strongly continuous semigroup. We also assume throughout that $\mu(z) \geq 0$ for almost all $z \in \partial \Omega$ so that the semigroup is positive.

For the definition of spectral bound and irreducibility we refer to Appendix A. The asymptotic behavior of $T_{\beta, \mu}^{C}$ is determined by the spectral bound $s\left(A_{\beta, \mu}^{C}\right)$ of its generator (see Appendix A). We first show that the spectrum is not empty.

Proposition 6.1. Assume that $\mu(z) \geq 0$ for almost all $z \in \partial \Omega$. Then $s\left(A_{\beta, \mu}^{C}\right)>$ $-\infty$. Moreover, $s\left(A_{\beta, \mu}^{C}\right)$ is an eigenvalue of $A_{\beta, \mu}^{C}$ with positive eigenfunction.

Proof. We first show that $s\left(A_{\beta, 0}^{C}\right) \leq s\left(A_{\beta, \mu}^{C}\right)$. As a consequence of Proposition 5.4 we have $0 \leq T_{\beta, 0}^{C}(t) \leq T_{\beta, \mu}^{C}(t)$. Taking Laplace transforms, it follows that $0 \leq R\left(\lambda, A_{\beta, 0}^{C}\right) \leq R\left(\lambda, A_{\beta, \mu}^{C}\right)$ for all large enough $\lambda$. By [6, Theorem 5.3.1] for a positive semigroup the abscissa of the Laplace transform coincides with the spectral bound. Thus, if we assume that $s\left(A_{\beta, 0}^{C}\right)>s\left(A_{\beta, \mu}^{C}\right)$ we have $0 \leq R\left(\lambda, A_{\beta, 0}^{C}\right) \leq R\left(\lambda, A_{\beta, \mu}^{C}\right)$ for all $\lambda>s\left(A_{\beta, 0}^{C}\right)$. By [6, Proposition 3.11.2] we have $s\left(A_{\beta, 0}^{C}\right) \in \sigma\left(A_{\beta, 0}^{C}\right)$ and hence $\sup _{\lambda>s\left(A_{\beta, 0}^{C}\right)}\left\|R\left(\lambda, A_{\beta, 0}^{C}\right)\right\|=\infty$. Consequently, also $\left\|R\left(\lambda, A_{\beta, \mu}^{C}\right)\right\|$ is unbounded as $\lambda \downarrow s\left(A_{\beta, 0}^{C}\right)$. It thus follows that $s\left(A_{\beta, 0}^{C}\right) \in \sigma\left(A_{\beta, \mu}^{C}\right)$, a contradiction to our assumption $s\left(A_{\beta_{0}}^{C}\right)>s\left(A_{\beta, \mu}^{C}\right)$.

The operator $A_{\beta, 0}^{C}$ is the part of $-\mathscr{A}_{\beta}^{2}$ in $C(\bar{\Omega})$, defined before Theorem 4.10. It follows from Proposition A.4 that the semigroup generated by $-\mathscr{A}_{\beta}^{2}$ is irreducible. Since 
the resolvent of that operator is compact, it follows from de Pagter's Theorem (see [16, Theorem 3] or [7, C-III Theorem 3.7.(c)]) that $s\left(-\mathscr{A}_{\beta}^{2}\right)>-\infty$. But we have $s\left(A_{\beta, 0}^{C}\right)=s\left(-\mathscr{A}_{\beta}^{2}\right)$ since the resolvents are compact and consistent, see [2, Proposition $2.6]$.

Note that the semigroup $T_{\beta, \mu}^{C}$ is compact and hence immediately norm continuous whence spectral bound and growth bound coincide. Thus, if $s\left(A_{\beta, \mu}\right)<0$, then $\left\|T_{\beta, \mu}^{C}(t)\right\| \leq M e^{-\varepsilon t}$ for all $t>0$ and suitable constants $M>0, \varepsilon>0$, i.e. the semigroup is exponentially stable. If, on the other hand, $s\left(A_{\beta, \mu}^{C}\right)>0$ then there exists $\varepsilon>0, M>0$ such that $\left\|T_{\beta, \mu}^{C}(t)\right\| \geq M e^{\varepsilon t}$ for all $t>0$. Finally, if $s\left(A_{\beta, \mu}\right)=0$, then the semigroup converges if it is bounded. This is not easy to decide, though. However, we have a precise criterion for the semigroup to be sub-Markovian. In that case, we obtain the following result from Theorem A.1.

Proposition 6.2. Assume that $\mu(z) \geq 0$ and

$$
\mu(z)(\bar{\Omega}) \leq \beta(z)+\sum_{j=1}^{d} \operatorname{tr} b_{j} \nu_{j}(z)
$$

for almost every $z \in \partial \Omega$ and

$$
\sum_{j=1}^{d} D_{j} b_{j} \leq d_{0}
$$

almost everywhere. Then there exist a positive projection $P \in \mathscr{L}(C(\bar{\Omega}))$ with finite rank and $M>0, \varepsilon>0$ such that

$$
\left\|T_{\mu, \beta}^{C}(t)-P\right\|_{\mathscr{L}(C(\bar{\Omega}))} \leq M e^{-\varepsilon t}
$$

for all $t>0$.

In the situation of Proposition 6.2, if $s\left(A_{\beta, \mu}^{C}\right)=0$, there exists a function $0<u=P u$, i.e. a positive function in the kernel of $A_{\beta, \mu}^{C}$. If the semigroup is Markovian, then $\mathbb{1}$ is such a function. It is interesting to know when it is the only one (up to a scalar multiple). If $T_{\beta, \mu}^{C}$ is irreducible, then this is the case. Unfortunately, it is not easy to prove irreducibility on $C(\bar{\Omega})$. However, it follows from the domination property $(5.5)$ that $T_{\beta, \mu}^{C}$ is irreducible whenever $T_{\beta, 0}^{C}$ is so. As for the latter semigroup, a particular case will be settled in Theorem 7.3. We also remark that in $[\mathbf{1 1}]$ it is shown that $T_{\beta, 0}^{C}$ is irreducible whenever $\Omega$ is connected, $b_{j}=0$ and $a_{i j}=a_{j i}$ for $i, j=1, \ldots, d$, see $[\mathbf{1 1}$, Section 2] and in particular Corollary 2.5 of that reference.

Theorem 6.3. Assume that $\mu(z) \geq 0$ and

$$
0 \leq \mu(z)(\bar{\Omega})=\beta(z)+\sum_{j=1}^{d} \operatorname{tr} b_{j} \nu_{j}(z)
$$


for almost all $z \in \partial \Omega$ and $\sum_{j=1}^{d} D_{j} b_{j}=d_{0}$. Assume further that $T_{\beta, 0}^{C}$ is irreducible. Then there exist a strictly positive measure $\rho$ on $\bar{\Omega}$ and constants $\varepsilon, M>0$ such that for $P \in \mathscr{L}(C(\bar{\Omega}))$, given by

$$
P f=\int_{\bar{\Omega}} f \mathrm{~d} \rho \cdot \mathbb{1}
$$

for all $f \in C(\bar{\Omega})$, we have

$$
\left\|T_{\beta, \mu}^{C}(t)-P\right\|_{\mathscr{L}(C(\bar{\Omega}))} \leq M e^{-\varepsilon t}
$$

for all $t>0$.

Proof. By Proposition 5.2 the semigroup $T_{\beta, \mu}^{C}$ is Markovian and hence $\mathbb{1}$ is a fixed vector of the semigroup. As a consequence of (5.5), $T_{\beta, \mu}^{C}$ is irreducible. Now the claim follows from Theorem A.2.

We next prove exponential stability in the sub-Markovian case.

TheOREM 6.4. Assume that $\mu(z) \geq 0$ for almost all $z \in \partial \Omega$ and that (6.1) and (6.2) hold. Moreover, assume that $T_{\beta, 0}^{C}$ is irreducible. If in (6.1) or (6.2) the inequality is strict on some set of positive measure, then there exist $\varepsilon, M>0$ such that

$$
\left\|T_{\beta, \mu}^{C}(t)\right\|_{\mathscr{L}(C(\bar{\Omega}))} \leq M e^{-\varepsilon t}
$$

for all $t>0$.

Proof. Let us put

$$
\tilde{\beta}(z):=\mu(z)(\bar{\Omega})-\sum_{j=1}^{d} \operatorname{tr} b_{j}(z) \nu_{j}(z)
$$

and $\tilde{d}_{0}(x)=\sum_{j=1}^{d}\left(D_{j} b_{j}\right)(x)$. Replace $d_{0}$ with $\tilde{d}_{0}$ and $\beta$ with $\tilde{\beta}$ and denote by $\tilde{T}_{\tilde{\beta}, \mu}$ the corresponding semigroup on $C(\bar{\Omega})$. We denote the generator of $\tilde{T}_{\tilde{\beta}, \mu}^{C}$ by $\tilde{A}_{\tilde{\beta}, \mu}^{C}$. Then $0 \leq T_{\beta, \mu}^{C}(t) \leq \tilde{T}_{\tilde{\beta}, \mu}^{C}(t)$ for all $t>0$ by Proposition 5.4 and a perturbation argument, cf. the proof of Proposition 5.5. By Proposition 5.3 the semigroup $\tilde{T}_{\tilde{\beta}, \mu}$ is Markovian so that its generator has spectral bound 0 . However, the generators of these two semigroups are different. To see this, let us first assume that $\beta \neq \tilde{\beta}$ in $L^{\infty}(\partial \Omega)$. Note that the conormal derivative $\partial_{\nu}^{\mathscr{A}}=\partial_{\mu}^{\tilde{A}}$ does not depend on the zero order term $d_{0}$ resp. $\tilde{d}_{0}$. We find

$$
\langle\mathbb{1}, \mu(z)\rangle=\partial_{\nu}^{\tilde{\mathscr{A}}} \mathbb{1}+\tilde{\beta} \mathbb{1} \neq \partial_{\nu}^{\mathscr{A}} \mathbb{1}+\beta \mathbb{1} .
$$

Thus $\mathbb{1} \notin D\left(A_{\beta, \mu}^{C}\right)$ but $\mathbb{1} \in D\left(\tilde{A}_{\beta, \mu}^{C}\right)$. If, on the other hand, $\beta=\tilde{\beta}$ in $L^{\infty}(\partial \Omega)$, then we have $d_{0} \neq \tilde{d}_{0}$ in $L^{\infty}(\Omega)$. Note that $A_{\beta, \mu} \mathbb{1}=\tilde{d}_{0}-d_{0}$. If $\tilde{d}_{0}-d_{0} \in C(\bar{\Omega})$, it follows that $\mathbb{1} \in D\left(A_{\beta, \mu}^{C}\right)$ but $A_{\beta, \mu}^{C} \mathbb{1} \neq \tilde{A}_{\tilde{\beta}, \mu}^{C} \mathbb{1}$. If $\tilde{d}_{0}-d_{0} \notin C(\bar{\Omega})$, then $\mathbb{1} \notin D\left(A_{\beta, \mu}^{C}\right)$. In any case we have $\tilde{A}_{\tilde{\beta}, \mu}^{C} \neq A_{\beta, \mu}^{C}$. Thus the claim follows from Theorem A.3. 
Next we show a blow-up result in the case where we perturb a Markovian semigroup $T_{\beta, 0}$ by a positive $\mu$. Recall from Proposition 4.5 that $T_{\beta, 0}$ is Markovian if and only if the identities (4.4) and (4.5) hold.

THEOREM 6.5. Assume the identities (4.4) and (4.5) and that $\Omega$ is connected. If $\mu(z) \geq 0$ for almost all $z \in \partial \Omega$ but not identically 0 almost everywhere, then there exist $\omega, M>0$ such that

$$
\left\|T_{\beta, \mu}^{C}(t)\right\|_{\mathscr{L}(C(\bar{\Omega}))} \geq M e^{\omega t}
$$

for all $t>0$.

Proof. The semigroup $T_{\beta, 0}^{C}$ is Markovian (by Proposition 4.5) and has an extension to $L^{2}(\Omega)$ which is irreducible (as a consequence of Proposition A.4). From Proposition A.5, it follows that $T_{\beta, 0}^{C}$ is irreducible. By Proposition 5.4 we have $T_{\beta, 0}^{C}(t) \leq T_{\beta, \mu}^{C}(t)$ for all $t>0$. Since $\partial_{\nu} \mathbb{1}+\beta \mathbb{1}=0<\mu(z)(\Omega)$ for $z$ in a set of positive measure, one has $\mathbb{1} \notin D\left(A_{\beta, \mu}^{C}\right)$. Thus the two semigroups are different and it follows from Theorem A.3 that $0=s\left(A_{\beta, 0}^{C}\right)<s\left(A_{\beta, \mu}^{C}\right)=: \omega$. Thus there exists $u \in C(\bar{\Omega})$ such that $u \geq \mathbb{1}$ with $A_{\beta, \mu}^{C} u=\omega u$. But this implies $T_{\beta, \mu}^{C}(t) u=e^{\omega t} u$ which, in turn, yields the claim.

REMARK 6.6. In particular, it follows from Theorem 6.5 that the only realization of our operator with non-local Neumann boundary conditions (i.e. where $\beta=0$ ) which generates a sub-Markovian semigroup is that with classical (local) Neumann boundary conditions (i.e. $\beta=0$ and $\mu=0$ ).

\section{Absolutely continuous measures $\mu(z)$.}

In this section we consider the case where the measures $\mu(z)$ are absolutely continuous with respect to the Lebesgue measure on $\Omega$. More precisely, we assume that we are given a function $h \in L^{2}(\partial \Omega \times \Omega)$ such that

$$
\mu(z)(A)=\int_{A} h(z, x) \mathrm{d} x .
$$

In this situation we can use form methods to show that the semigroup $T_{\beta, \mu}$, defined on $L^{\infty}(\Omega)$, has an extension to $L^{2}(\Omega)$. This allows us to establish irreducibility of $T_{\beta, \mu}^{C}$ via Propositions A.4 and A.5 in the Markovian case, provided $\Omega$ is connected. On the other hand, we can use form methods to show that our assumptions to infer positivity resp. sub-Markovianity are close to optimal.

We consider the form $\mathfrak{a}_{\beta, h}: H^{1}(\Omega) \times H^{1}(\Omega) \rightarrow \mathbb{R}$, given by

$$
\mathfrak{a}_{\beta, h}[u, v]:=\mathfrak{a}_{\beta}[u, v]-\int_{\partial \Omega} \int_{\Omega} h(z, x) u(x) \mathrm{d} x v(z) \mathrm{d} \sigma(z) .
$$

Then the form $\mathfrak{a}_{\beta, h}$ is elliptic and continuous. Denote by $\mathscr{A}_{\beta, h}^{2}$ the associated operator on $L^{2}(\Omega)$. Then $-\mathscr{A}_{\beta, h}^{2}$ generates a holomorphic, strongly continuous semigroup $T_{\beta, h}^{2}$ on $L^{2}(\Omega)$. It is easy to see that if in addition 


$$
\int_{\partial \Omega}\left(\int_{\Omega}|h(z, x)| \mathrm{d} x\right)^{p} \mathrm{~d} \sigma
$$

for some $p>d-1$ with $p \geq 2$, then the measures $\mu(z)=h(z, x) \mathrm{d} x$ satisfy Hypothesis 1.1 whence we obtain a semigroup $T_{\beta, \mu}$ on $L^{\infty}(\Omega)$ with generator $A_{\beta, \mu}$. Using the definition of the co-normal derivative one sees that the part of $-\mathscr{A}_{\beta, h}^{2}$ in $L^{\infty}(\Omega)$ is precisely the operator $A_{\beta, \mu}$. It follows that $T_{\beta, h}^{2}$ leaves the space $L^{\infty}(\Omega)$ invariant and the restriction of that semigroup to $L^{\infty}(\Omega)$ is $T_{\beta, \mu}$.

Proposition 7.1. With the notation above, we have:

(a) The semigroup $T_{\beta, h}^{2}$ is positive if and only if $h \geq 0$ almost everywhere.

(b) Assume that $b_{j} \in W^{1, \infty}(\Omega)$ for $j=1, \ldots, d$. Then $T_{\beta, h}^{2}$ is sub-Markovian if and only if (5.3) holds, $h \geq 0$ almost everywhere and $0 \leq \int_{\Omega} h(z, x) \mathrm{d} x \leq \beta(z)+$ $\sum_{j=1}^{d} \operatorname{tr} b_{j}(z) \nu_{j}(z)$ for almost every $z \in \partial \Omega$.

Proof. (a) By the first Beurling-Deny criterion [29, Corollary 2.6] $T_{\beta, \mu}^{2}$ is positive if and only if $\mathfrak{a}_{\beta, \mu}\left[u^{+}, u^{-}\right] \leq 0$ for all $u \in H^{1}(\Omega)$. If $h \geq 0$ almost everywhere this is clearly fulfilled.

Conversely assume that $T_{\beta, \mu}^{2}(t) \geq 0$ for all $t>0$. Then

$$
\int_{\partial \Omega} \int_{\Omega} h(z, x) u^{+}(x) \mathrm{d} x u^{-}(z) \mathrm{d} \sigma(z)=-\mathfrak{a}_{\beta, h}\left[u^{+}, u^{-}\right] \geq 0
$$

for all $u \in H^{1}(\Omega)$. Now let functions $0 \leq v \in \mathscr{D}(\Omega)$ and $0 \leq \varphi \in C(\partial \Omega)$ be given. We find a sequence $w_{n} \in \mathscr{D}\left(\mathbb{R}^{d}\right)$ with $0 \leq w_{n} \leq\|\varphi\|_{\infty}$ such that $\operatorname{supp} w_{n} \cap \operatorname{supp} v=\emptyset$ and $w_{n}(z) \rightarrow \varphi(z)$ for all $z \in \partial \Omega$. Inserting $u=v-w_{n}$ in the above inequality and using dominated convergence, we obtain that

$$
\int_{\partial \Omega} \int_{\Omega} h(z, x) v(x) \mathrm{d} x \varphi(z) \mathrm{d} \sigma(z) \geq 0 .
$$

As $0 \leq \varphi \in C(\partial \Omega)$ was arbitrary, we conclude that

$$
\int_{\Omega} h(z, x) v(x) \mathrm{d} x \geq 0
$$

for almost all $z \in \partial \Omega$. As $0 \leq v \in \mathscr{D}(\Omega)$ was arbitrary, it follows that for almost all $z \in \partial \Omega$ we have $h(z, x)=0$ for almost all $x \in \Omega$. Now Fubini's theorem implies that $h \geq 0$ with respect to the product measure, proving the necessity of the condition.

(b) The sufficiency of the inequality above was already established in Proposition 5.5 , so we only need to prove its necessity. If the semigroup is sub-Markovian, it is positive and thus $h \geq 0$ almost everywhere by (a).

By the Beurling-Deny-Ouhabaz criterion [29, Corollary 2.8], for $u \in H^{1}(\Omega)$ we have

$$
0 \leq \mathfrak{a}_{\beta, h}\left[u \wedge 1,(u-1)^{+}\right]
$$




$$
\begin{aligned}
= & -\sum_{j} \int_{\Omega}\left(D_{j} b_{j}\right)(u-1)^{+} \mathrm{d} x+\int_{\Omega} d_{0}(u-1)^{+} \mathrm{d} x \\
& +\int_{\partial \Omega}\left(\sum_{j} b_{j} \nu_{j}(u-1)^{+}+\beta(z)-\int_{\Omega}(u \wedge 1)(x) h(z, x) \mathrm{d} x\right)(u-1)^{+}(z) \mathrm{d} \sigma(z) .
\end{aligned}
$$

Now let $v \in H^{1}(\Omega)$ such that $v \geq 0$. Inserting $u=v+\mathbb{1}$ in the above inequality, the desired inequalities follow from Lemma 4.4.

Remark 7.2. We have already noted after Proposition 5.5 that Condition (5.3) is necessary for $T_{\beta, \mu}$ to be sub-Markovian.

We now consider the case where the semigroup is Markovian. Then we can prove irreducibility via Proposition A.4 and deduce convergence of the semigroup to an equilibrium.

TheOREM 7.3. Assume that $\Omega$ is connected, and that $h \geq 0$ almost everywhere satisfies Equation (7.1). Moreover, assume that $\sum_{j=1}^{d} D_{j} b_{j}=d_{0}$ almost everywhere on $\Omega$ and

$$
\sum_{j=1}^{d} b_{j}(z) \nu_{j}(z)+\beta(z)=\int_{\Omega} h(z, x) \mathrm{d} x
$$

almost everywhere on $\partial \Omega$. Then the semigroup $T_{\beta, \mu}^{C}$ on $C(\bar{\Omega})$ is irreducible and Markovian. Consequently, there exist $0 \ll \varphi \in L^{2}(\Omega)$ such that $\int_{\Omega} \varphi(x) \mathrm{d} x=1$ and constants $\varepsilon, M>0$ such that

$$
\left\|T_{\beta, \mu}^{C}(t)-\varphi \otimes \mathbb{1}\right\|_{\mathscr{L}(C(\bar{\Omega}))} \leq M e^{-\varepsilon t}
$$

for all $t>0$.

\section{Measures satisfying Hypothesis 1.1.}

In this brief section we give some examples of maps $\mu$ for which Hypothesis 1.1 is satisfied.

ExAmple 8.1. Assume that for every Borel set $A \subset \bar{\Omega}$ the complex-valued map $z \mapsto \mu(z)(A)$ is continuous. Then $\mu$ satisfies conditions (a), (b) and (c) in Hypothesis 1.1.

Proof. It is obvious that (a) holds. As for (b), we note that by continuity and compactness of $\partial \Omega$ we have $\sup _{z \in \partial \Omega}|\mu(z)(A)|<\infty$ for every $A \in \mathscr{B}(\bar{\Omega})$. Now [12, Corollary 4.6.4] yields $\sup _{z \in \partial \Omega}\|\mu(z)\|<\infty$. To prove (c), pick a dense sequence $z_{n}$ in $\partial \Omega$. We set

$$
\tau:=\sum_{n \in \mathbb{N}} \frac{1}{2^{n}}\left|\mu\left(z_{n}\right)\right|,
$$

where $|\mu(z)|$ denotes the total variation of $\mu(z)$. Then $\tau$ is a finite positive measure and 
we have $\mu\left(z_{n}\right) \ll \tau$ for every $n \in \mathbb{N}$. Let $A \in \mathscr{B}(\bar{\Omega})$ with $\tau(A)=0$ be given. Consider the function $\varphi(z):=\mu(z)(A)$. By the above $\varphi\left(z_{n}\right)=0$ for all $n \in \mathbb{N}$. Moreover, $\varphi$ is continuous by assumption. Thus $\varphi \equiv 0$, proving that in fact $\mu(z) \ll \tau$ for all $z \in \partial \Omega$.

Similarly, we can consider maps $\mu$ which only take countably many values.

EXAMPLE 8.2. Assume that $\mu(z)=\sum_{n \in J} \mathbb{1}_{A_{n}}(z) \mu_{j}$ where $\left(A_{n}\right)_{j \in J} \subset \mathscr{B}(\partial \Omega)$ and $\left(\mu_{j}\right)_{n \in J} \subset \mathscr{M}(\bar{\Omega})$ and $J$ is a finite or countably infinite index set. Then $\mu$ satisfies Hypothesis 1.1 provided $\sum_{n \in J} \sigma\left(A_{n}\right)\left|\mu_{n}\right|(\bar{\Omega})^{p}<\infty$ where $p$ is as in Hypothesis 1.1(b).

Proof. Part (a) is obvious and (b) was assumed. Part (c) is fulfilled with $\tau=$ $\sum_{n \in J} 2^{-n}\left|\mu_{n}\right|$.

\section{Appendix A. Irreducible semigroups.}

In this appendix we collect some known facts on positive, irreducible semigroups. In some cases we present some variations or adapt results to our special situation.

Let $E$ be a real Banach lattice. In our context $E$ will be $C(\bar{\Omega})$ or $L^{q}(\Omega)$. Let $T$ be a strongly continuous semigroup on $E$ which is positive, i.e. for $f \in E_{+}$we have $T(t) f \in E_{+}$for all $t \geq 0$. We denote the generator of $T$ by $A$. The spectral bound of $A$ is defined by

$$
s(A):=\sup \left\{\operatorname{Re} \lambda: \lambda \in \sigma\left(A_{\mathbb{C}}\right)\right\}
$$

where $\sigma\left(A_{\mathbb{C}}\right)$ is the spectrum of the generator $A_{\mathbb{C}}$ of the complexification of $T$. In what follows, we will not distinguish between an operator and its complexification. In particular, when we talk about the spectrum, resolvent, etc. of an operator, we always mean the spectrum/resolvent, etc. of its complexification.

By [7, C-III Theorem 1.1], $s(A) \in \sigma(A)$ whenever $\sigma(A) \neq \emptyset$. If $A$ has compact resolvent, then $\sigma(A)$ consists of isolated points which are all eigenvalues.

TheOREM A.1. Assume that $T(t)$ is compact for all $t>0$, that $s(A)=0$ and that $T$ is bounded. Then there exist a positive projection $P \neq 0$ of finite rank, $\varepsilon>0$ and $M>0$ such that

$$
\|T(t)-P\|_{\mathscr{L}(E)} \leq M e^{-\varepsilon t}
$$

for all $t>0$.

Proof. Since $T(t)$ is compact for all $t>0, T$ is immediately norm continuous and it follows from [7, C-III Corollary 2.13] that there is some $\delta>0$ such that $\operatorname{Re} \lambda \leq-2 \delta<0$ for all $\lambda \in \sigma(A) \backslash\{0\}$. Denote by $P$ the spectral projection with respect to 0 , i.e.

$$
P:=\frac{1}{2 \pi i} \int_{|\lambda|=\delta} R(\lambda, A) \mathrm{d} \lambda .
$$


As $T(t)$ is compact for all $t>0$, so is the resolvent and thus also $P$, whence it has finite rank. The restriction of $T$ to the range of $P$ is a bounded semigroup on a finite dimensional vector space whose generator has spectrum $\{0\}$. It follows that the generator of the restriction is diagonalizable and is thus the zero operator. Consequently, $T(t) P=P$ for all $t>0$. The space $F=(I-P) E$ is invariant under the semigroup and the generator $A_{F}$ of the restriction has its spectrum in a strict left half plane. Since the semigroup is immediately norm continuous there exist $\varepsilon>0, M>0$ such that $\left\|\left.T(t)\right|_{F}\right\|_{\mathscr{L}(F)} \leq M e^{-\varepsilon t}$ and hence $\|T(t)-P\|_{\mathscr{L}(E)} \leq M e^{-\varepsilon t}$ for all $t \geq 0$.

Theorem A.1 implies in particular that there exists $u>0$, i.e. $u \geq 0$ and $u \neq 0$, such that $T(t) u=u$ for all $t \geq 0$. Thus the Krein-Rutman Theorem which asserts that the largest eigenvalue (i.e. $s(A)$ ) has a positive eigenfunction is incorporated in Theorem A.1.

We next want to investigate when $P$ has rank one and the positive eigenfunction is strictly positive. This will be done via the notion of irreducibility. A subspace $J$ of $E$ is called an ideal if

(i) $u \in J$ implies $|u| \in J$ and

(ii) if $u \in J$, then $0 \leq v \leq u$ implies $v \in J$.

A positive, strongly continuous semigroup $T$ on $E$ is called irreducible if the only invariant closed ideals are $J=\{0\}$ and $J=E$.

If $E=C(\bar{\Omega})$ then $J \subset E$ is a closed ideal if and only if there exists a closed subset $K$ of $\bar{\Omega}$ such that

$$
J=\left\{f \in C(\bar{\Omega}):\left.f\right|_{K}=0\right\} .
$$

If $E=L^{q}(\Omega)(1 \leq q<\infty)$ then $J \subset E$ is a closed ideal if and only if there exists a measurable subset $K$ of $\Omega$ such that

$$
J=\left\{f \in L^{q}(\Omega):\left.f\right|_{K}=0 \text { a.e. }\right\} .
$$

We say that $u \in E$ is a quasi interior point and write $u \gg 0$ if the principal ideal

$$
E_{u}:=\{v \in E: \exists c>0 \text { such that }|v| \leq c u\}
$$

is dense in $E$.

If $E=C(\bar{\Omega})$ then $u \gg 0$ if and only if there is $\delta>0$ such that $u(x) \geq \delta>0$ for all $x \in \bar{\Omega}$. In this case $u$ is actually an inner point of the positive cone. If $E=L^{p}(\Omega)$ then $u \gg 0$ if and only if $u(x)>0$ for almost every $x$.

We call $\varphi \in E^{\prime}$ a strictly positive functional if $\langle\varphi, f\rangle=0$ implies $f=0$ for all $f \in E_{+}$.

If $E=C(\bar{\Omega})$, then $\varphi$ is strictly positive if and only if there exists a strictly positive Borel measure $\nu$, i.e. $\nu(O)>0$ for all non-empty open sets $O \subset \bar{\Omega}$, such that

$$
\langle\varphi, f\rangle=\int_{\bar{\Omega}} f(x) \mathrm{d} \nu(x) .
$$


If $E=L^{q}(\Omega)$ for $\varphi \in L^{q^{\prime}}(\Omega) \simeq\left(L^{q}(\Omega)\right)^{\prime}$ to be strictly positive is equivalent to that $\varphi(x)>0$ almost everywhere, i.e. $\varphi \gg 0$.

The importance of these concepts in the study of asymptotic behavior stems from the fact that positive fixed points of positive, irreducible semigroups are strictly positive. More precisely, if $T$ is a positive, irreducible, strongly continuous semigroup and $u>0$ is such that $T(t) u=u$ for all $t>0$, then $u \gg 0$ and if $0<\varphi \in E^{\prime}$ is such that $T(t)^{\prime} \varphi=\varphi$ for all $t>0$ then $\varphi$ is strictly positive. Moreover, because of irreducibility, $s(A)$ cannot be a pole of order larger than 1, see [7, C-III Proposition 3.5]. This implies that $T(t) P=P$ for all $t>0$ in the proof of Theorem A.1 even though the semigroup is not assumed to be bounded. We thus obtain the following result on asymptotic stability.

THEOREM A.2. Let $T$ be a positive, irreducible strongly continuous semigroup on $E$ with generator $A$. Assume that $T(t)$ is compact for $t>0$ and $s(A)=0$. Then there exist $0 \ll u \in \operatorname{ker} A$, a strictly positive $\varphi \in \operatorname{ker} A^{\prime}, \varepsilon>0, M>0$ such that $\langle\varphi, u\rangle=1$ and

$$
\|T(t)-\varphi \otimes u\|_{\mathscr{L}(E)} \leq M^{-\varepsilon t}
$$

for all $t \geq 0$ where we have written $\varphi \otimes u$ for the projection defined by

$$
(\varphi \otimes u)(f)=\langle\varphi, f\rangle u
$$

for all $f \in E$. In particular

$$
\lim _{t \rightarrow \infty} T(t) f=\langle\varphi, f\rangle u
$$

i.e. the orbits of the semigroup converge to an equilibrium.

Theorems A.1 and A.2 lie at the heart of the Perron-Frobenius theory. We refer to [7] for more information.

We shall have occasion to use the strict monotonicity of the spectral bound.

THEOREM A.3. Let $S$ and $T$ be strongly continuous semigroups on $E$ with generators $B$ and $A$ respectively. Assume that

(i) $0 \leq S(t) \leq T(t)$ for all $t>0$;

(ii) A has compact resolvent, and

(iii) $T$ is irreducible.

If $A \neq B$, then $s(B)<s(A)$.

Proof. This is a version of [5, Theorem 1.3], see also [4, Theorem 10.2.10] in connection with [4, Theorems 10.6.3 and 10.6.1].

Next we describe ways to prove irreducibility. On $L^{2}(\Omega)$ this is very easy if the semigroup is associated with a form by virtue of the Beurling-Deny-Ouhabaz criterion for the invariance of closed convex sets. In particular the following holds true (see [31, Theorem 2.10]). 
Proposition A.4. Let $V \subset H^{1}(\Omega)$ be a closed subspace containing $H_{0}^{1}(\Omega)$, where $\Omega \subset \mathbb{R}^{d}$ is a connected, open set. Let $\mathfrak{a}: V \times V \rightarrow \mathbb{R}$ be a continuous and elliptic form such that the associated semigroup $T$ is positive. Then $T$ is irreducible.

On $C(\bar{\Omega})$ irreducibility is a stronger notion than on $L^{2}(\Omega)$. However, the following result shows how irreducibility on $C(\bar{\Omega})$ can be deduced from irreducibility on $L^{2}(\Omega)$.

Proposition A.5. Let $\Omega \subset \mathbb{R}^{d}$ be open and bounded and $T$ be a positive, irreducible, strongly continuous semigroup on $L^{2}(\Omega)$ whose generator $A$ has compact resolvent. Assume that $T$ leaves $C(\bar{\Omega})$ invariant and that the restriction $T^{C}$ of $T$ to $C(\bar{\Omega})$ is strongly continuous and suppose that its generator $A^{C}$ has compact resolvent. Assume that $s(A)=0$. Then $T^{C}$ is irreducible if and only if there exists $u \in \operatorname{ker} A \cap C(\bar{\Omega})$ such that $u(x) \geq \delta>0$ for all $x \in \bar{\Omega}$.

Proof. Assume that there exists $0 \ll u \in C(\bar{\Omega}) \cap \operatorname{ker} A$. Since $T$ is irreducible 0 is a pole of order 1 and the residuum $P$ is of the form

$$
P f=\left(\int_{\Omega} \varphi f \mathrm{~d} x\right) \cdot u
$$

for some $0 \ll \varphi \in L^{2}(\Omega)$, see [7, C-III Proposition 3.5]. Since $C(\bar{\Omega})$ is dense in $L^{2}(\Omega)$, it follows that the coefficients in the Laurent series expansion in $C(\bar{\Omega})$ around 0 (see [7, A-III, Equation (3.1)]) are the restriction of those in $L^{2}(\Omega)$. Thus 0 is also a pole of order 1 of the resolvent of $A^{C}$. The residuum

$$
P^{C}=\frac{1}{2 \pi i} \int_{|\lambda|=\varepsilon} R\left(\lambda, A^{C}\right) \mathrm{d} \lambda
$$

is the same, i.e. $P^{C}=\left.P\right|_{C(\bar{\Omega})}$. Now let $J=\left\{f \in C(\bar{\Omega}):\left.f\right|_{K}=0\right\}$ be an invariant ideal. Then for $z \in K, f \in J, f \geq 0$ we have $(T(t) f)(z)=0$ for all $t>0$ and hence $\left(R\left(\lambda, A^{C}\right) f\right)(z)=0$ for all $\lambda>0$, since we suppose that $s(A)=0$ and know that $s(A)$ is the abscissis of the Laplace transform of the semigroup [6, Theorem 5.3.1]. Thus

$$
\int_{\Omega} f(x) \varphi(x) \mathrm{d} x \cdot u(z)=\lim _{\lambda \downarrow 0}\left(\lambda R\left(\lambda, A^{C}\right) f\right)(z)=0 .
$$

Since $\varphi \gg 0$ in $L^{2}(\Omega)$ this implies $f=0$. Consequently $J=\{0\}$. This proves the sufficiency.

To show the necessity, recall that 0 is also a pole of $R\left(\lambda, A^{C}\right)$. It follows that $s\left(A^{C}\right)=0$. By Theorem A.2, there exists $0 \ll u \in \operatorname{ker}\left(A^{C}\right) \subset \operatorname{ker}(A)$.

Acknowledgements. The authors are grateful to Jochen Glück for discussions on a train from Ulm to Munich which led to Proposition A.5.

\section{References}

[1] M. S. Agranovich, Sobolev spaces, their generalizations and elliptic problems in smooth and Lipschitz domains, Revised translation of the 2013 Russian original, Springer Monographs in Math., Springer, Cham, 2015. 
[2] W. Arendt, Gaussian estimates and interpolation of the spectrum in $L^{p}$, Differential Integral Equations, 7 (1994), 1153-1168.

[ 3 ] W. Arendt, Semigroups and evolution equations: Functional calculus, regularity and kernel estimates, vol. 1 of Handbook of Differential Equations: Evolutionary Equations, North-Holland, 2002, 1-85.

[4] W. Arendt, Heat kernels, lecture notes of the 9th internet seminar, 2005.

[5] W. Arendt and C. J. K. Batty, Domination and ergodicity for positive semigroups, Proc. Amer. Math. Soc., 114 (1992), 743-747.

[6] W. Arendt, C. J. K. Batty, M. Hieber and F. Neubrander, Vector-valued Laplace transforms and Cauchy problems, Monographs in Mathematics, 96, Birkhäuser/Springer Basel AG, Basel, second ed., 2011.

[ 7 ] W. Arendt, A. Grabosch, G. Greiner, U. Groh, H. P. Lotz, U. Moustakas, R. Nagel, F. Neubrander and U. Schlotterbeck, One-parameter semigroups of positive operators, Lecture Notes in Math., 1184, Springer-Verlag, Berlin, 1986.

[8] W. Arendt, S. Kunkel and M. Kunze, Diffusion with nonlocal boundary conditions, J. Funct. Anal., 270 (2016), 2483-2507.

[ 9 ] W. Arendt and R. Mazzeo, Spectral properties of the dirichlet-to-neumann operator, Ulmer Seminare, 12 (2007), 23-37.

[10] W. Arendt and A. F. M. ter Elst, Gaussian estimates for second order elliptic operators with boundary conditions, J. Operator Theory, 38 (1997), 87-130.

[11] W. Arendt and A. F. M. ter Elst, The Dirichlet-to-Neumann operator on $C(\partial \Omega)$, preprint, 2017.

[12] V. I. Bogachev, Measure theory, Vol. I, II, Springer-Verlag, Berlin, 2007.

[13] D. M. Bošković, M. Krstić and W. Liu, Boundary control of an unstable heat equation via measurement of domain-averaged temperature, IEEE Trans. Automat. Control, 46 (2001), 2022-2028.

[14] D. Daners, Heat kernel estimates for operators with boundary conditions, Math. Nachr., 217 (2000), 13-41.

[15] D. Daners, Inverse positivity for general Robin problems on Lipschitz domains, Arch. Math. (Basel), 92 (2009), 57-69.

[16] B. de Pagter, Irreducible compact operators, Math. Z., 192 (1986), 149-153.

[17] D. Dier, Non-autonomous forms and invariance, preprint, available at arXiv:1609.03857, 2016.

[18] K.-J. Engel and R. Nagel, One-parameter semigroups for linear evolution equations, Graduate Texts in Math., 194, Springer-Verlag, New York, 2000.

[19] W. Feller, The parabolic differential equations and the associated semi-groups of transformations, Ann. of Math. (2), 55 (1952), 468-519.

[20] W. Feller, Diffusion processes in one dimension, Trans. Amer. Math. Soc., 77 (1954), 1-31.

[21] W. Feller, Generalized second order differential operators and their lateral conditions, Illinois J. Math., 1 (1957), 459-504.

[22] E. I. Galakhov and A. L. Skubachevskiǔ, On Feller semigroups generated by elliptic operators with integro-differential boundary conditions, J. Differential Equations, 176 (2001), 315-355.

[23] G. Greiner, Perturbing the boundary conditions of a generator, Houston J. Math., 13 (1987), 213-229.

[24] P. L. Gurevich, W. Jäger and A. L. Skubachevskiı̌, On the existence of periodic solutions of some nonlinear problems of thermal control, Dokl. Akad. Nauk, 418 (2008), 151-154.

[25] M. Kunze, A Pettis-type integral and applications to transition semigroups, Czechoslovak Math. J., 61 (2011), 437-459.

[26] M. Kunze, Perturbation of strong Feller semigroups and well-posedness of semilinear stochastic equations on Banach spaces, Stochastics, 85 (2013), 960-986.

[27] H. P. Lotz, Uniform convergence of operators on $L^{\infty}$ and similar spaces, Math. Z., 190 (1985), 207-220.

[28] A. Lunardi, Analytic semigroups and optimal regularity in parabolic problems, Progress in Nonlinear Differential Equations and their Applications, 16, Birkhäuser Verlag, Basel, 1995.

[29] A. Manavi, H. Vogt and J. Voigt, Domination of semigroups associated with sectorial forms, J. Operator Theory, 54 (2005), 9-25.

[30] R. Nittka, Regularity of solutions of linear second order elliptic and parabolic boundary value 
problems on Lipschitz domains, J. Differential Equations, 251 (2011), 860-880.

[31] E. M. Ouhabaz, Analysis of heat equations on domains, London Math. Soc. Monographs Series, 31, Princeton University Press, Princeton, NJ, 2005.

[32] K. Sato and T. Ueno Multi-dimensional diffusion and the Markov process on the boundary, J. Math. Kyoto Univ., 4 (1964/1965), 529-605.

[33] A. L. Skubachevskir, Some problems for multidimensional diffusion processes, Dokl. Akad. Nauk SSSR, 307 (1989), 287-291. translation in Soviet Math. Dokl., 40 (1990), 75-79.

[34] A. L. Skubachevskiı̌, Nonlocal elliptic problems and multidimensional diffusion processes, Russian J. Math. Phys., 3 (1995), 327-360.

[35] K. Taira, Semigroups, boundary value problems and Markov processes, Springer Monographs in Math., Springer, Heidelberg, second ed., 2014.

[36] K. Taira, Analytic semigroups and semilinear initial boundary value problems, London Math. Soc. Lecture Note Series, 434, Cambridge University Press, Cambridge, second ed., 2016.

[37] A. D. Venttsel', On boundary conditions for multi-dimensional diffusion processes, Theor. Probability Appl., 4 (1959), 164-177.

\section{Wolfgang ARENDT}

Institute of Applied Analysis

Ulm University

89069 Ulm, Germany

E-mail: wolfgang.arendt@uni-ulm.de

\section{Stefan KunKEL}

Graduiertenkolleg 1100

Ulm University

89069 Ulm, Germany

E-mail: stefan.kunkel@uni-ulm.de

\section{Markus KunZE}

Universität Konstanz

Fachbereich Mathematik und Statistik

78467 Konstanz, Germany

E-mail: markus.kunze@uni-konstanz.de 